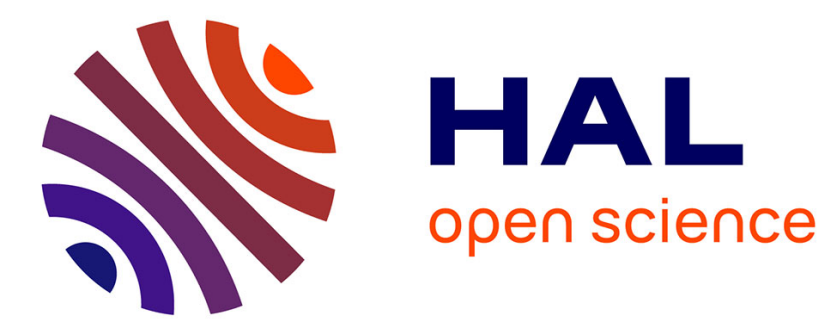

\title{
Dynamiques symplectiques génériques
}

Marie-Claude Arnaud, Christian Bonatti, Sylvain Crovisier

\section{To cite this version:}

Marie-Claude Arnaud, Christian Bonatti, Sylvain Crovisier. Dynamiques symplectiques génériques. Ergodic Theory and Dynamical Systems, 2005, 25 (5), pp.1401-1436. 10.1017/S0143385704000975. hal-00538131

\section{HAL Id: hal-00538131 \\ https://hal.science/hal-00538131}

Submitted on 21 Nov 2010

HAL is a multi-disciplinary open access archive for the deposit and dissemination of scientific research documents, whether they are published or not. The documents may come from teaching and research institutions in France or abroad, or from public or private research centers.
L'archive ouverte pluridisciplinaire HAL, est destinée au dépôt et à la diffusion de documents scientifiques de niveau recherche, publiés ou non, émanant des établissements d'enseignement et de recherche français ou étrangers, des laboratoires publics ou privés. 


\title{
Dynamiques symplectiques génériques
}

\author{
Marie-Claude Arnaud, Christian Bonatti et Sylvain Crovisier
}

22 novembre 2010

\begin{abstract}
Résumé
Nous montrons que $C^{1}$-génériquement les difféomorphismes symplectiques d'une variété compacte sont transitifs. Sur les variétés symplectiques non-compactes, nous montrons que les orbites génériques d'un difféomorphisme générique ne sont pas bornées. L'outil principal est un lemme de connexion pour les pseudo-orbites des difféomorphismes symplectiques. Pour cela nous donnons des conditions générales sur un espace de difféomorphismes, en particulier au voisinage des orbites périodiques, qui permettent d'obtenir le lemme de connexion pour les pseudo-orbites.
\end{abstract}

\begin{abstract}
We prove that $C^{1}$-generic symplectic diffeomorphisms of a compact manifold are transitive. On non-compact manifolds, we show that generic orbits of generic diffeomorphisms are not bounded. The main tool is a connecting lemma for pseudo-orbits of symplectic diffeomorphisms. In the proof, we give general conditions on diffeomorphisms spaces, in particular at the neighborhood of the periodic orbits, which imply the connecting lemma for pseudo-orbits.
\end{abstract}

\section{Table des matières}

\section{Introduction}

2 Perturbations locales $\quad 7$

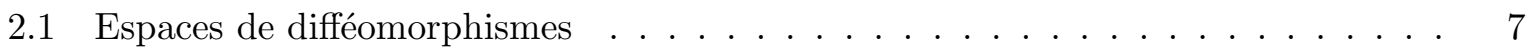

2.2 Domaines quadrillés, boîtes de perturbation . . . . . . . . . . . . 8

2.3 Existence de boîtes de perturbation : le connecting lemma . . . . . . . . . . . . 9

3 Perturbations globales $\quad 10$

3.1 Familles de boîtes couvrantes . . . . . . . . . . . . . . . . . . . 10

3.2 Existence de familles de boîtes couvrantes, loin des orbites périodiques de basse période . . . . . . . . . . . . . . . . . . 13

3.3 Perturbations au voisinage des orbites périodiques f . . . . . . . . . . . . 14

4 Au voisinage des points périodiques d'un difféomorphisme symplectique $\quad \mathbf{1 7}$

4.1 Généralités sur les points périodiques des difféomorphismes symplectiques . . . . 18

4.2 Linéarisation du problème de contournabilité d'une orbite périodique . . . . . . . 19

4.3 Familles couvrantes de cubes quadrillés . . . . . . . . . . . . . . . . . 21 


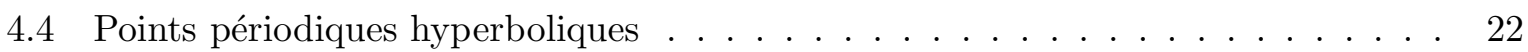

4.5 Points périodiques complètement elliptiques . . . . . . . . . . . . . . 23

4.6 Points périodiques elliptiques (cas général) . . . . . . . . . . . . . . . 24

5 Démonstration des résultats principaux 28

5.1 Résultats de généricité dans le cas compact . . . . . . . . . . . . . . 28

5.2 Résultats de généricité dans le cas non compact . . . . . . . . . . . . . . . . . 31

\section{Introduction}

Soit $(M, \omega)$ une variété symplectique compacte et $f$ un difféomorphisme symplectique de $M$. Le théorème de récurrence de Poincaré montre que l'ensemble non-errant de $f$ est la variété $M$ toute entière. Si $M$ est connexe, $f$ est transitif par chaînes, c'est à dire que l'on peut joindre deux points quelconques de $M$ par des pseudo-orbites de sauts arbitrairement petits. Il est alors naturel de se demander si l'on peut perturber $f$ (parmi les difféomorphismes symplectiques) de manière à le rendre transitif.

Remarquons que cette question se pose également pour les difféomorphismes conservatifs d'une variété compacte $M$ munie d'une forme volume $v$. Dans ce cas, la réponse est connue partiellement :

- Oxtoby et Ulam ont montré dans $[\mathrm{OU}]$ que les homéomorphismes conservatifs génériques d'une variété compacte connexe $(M, v)$ sont ergodiques et donc transitifs.

- Plus récemment, $[\mathrm{BC}]$ montre que tout difféomorphisme conservatif d'une variété compacte connexe $(M, v)$ peut être perturbé en topologie $C^{1}$ pour devenir transitif (les difféomorphismes conservatifs $C^{1}$-génériques sont donc transitifs).

On sait aussi qu'en topologie $C^{k}$ pour $k \geq 3$ et $\operatorname{dim} M=2$, la réponse est en général non : ceci est dû à l'existence persistante de tores K.A.M. invariants (voir $[\mathrm{H}]$ ). Quand $d=$ $\operatorname{dim} M>2$, ceci est encore vrai en grande différentiabilité : les théorèmes K.A.M. s'appliquent et permettent de montrer l'existence de tores de codimension 1 invariants pour des ouverts $C^{\infty}$ de difféomorphismes (voir [Y, Section 4.6.3]), pouvant briser $C^{\infty}$-robustement la transitivité.

Dans cet article, nous appliquons la méthode développée dans [BC] à l'ensemble $\operatorname{Diff}_{\omega}^{1}(M)$ des difféomorphismes symplectiques de classe $C^{1}$ de $M$, et montrons :

Théorème 1. Soit $(M, \omega)$ une variété compacte connexe symplectique. L'ensemble $\mathcal{G}$ des difféomorphismes $f \in \operatorname{Diff}_{\omega}^{1}(M)$ qui sont transitifs est un $G_{\delta}$ dense de $\operatorname{Diff}_{\omega}^{1}(M)$. De plus, l'ensemble $\mathcal{G}^{\prime} \subset \mathcal{G}$ des difféomorphismes pour lesquels $M$ est une unique classe homocline contient un $G_{\delta}$ dense de $\operatorname{Diff}_{\omega}^{1}(M)$.

Remarques 1.1. 1. Pour toute variété compacte connexe symplectique $(M, \omega)$, l'ensemble $\mathcal{G}$ des difféomorphismes symplectiques transitifs ne contient pas d'ouvert dense dans $\operatorname{Diff}_{\omega}^{1}(M)$. En effet, il existe un ouvert $\mathcal{O}$ non vide dans $\operatorname{Diff}_{\omega}^{1}(M)$ (en fait dans chaque classe d'isotopie de Diff $\left.{ }_{\omega}^{1}(M)\right)$ dans lequel les difféomorphismes non transitifs sont denses. Démonstration : Soit $f \in \operatorname{Diff}_{\omega}^{1}(M)$ un difféomorphisme ayant un point périodique $p_{f}$ complètement elliptique dont les valeurs propres (qui sont toutes de module 1) sont simples. Il existe alors un voisinage ouvert $\mathcal{O}_{f}$ de $f$ sur lequel la continuation de $p_{f}$ existe et est complètement elliptique. Tout difféomorphisme de $\mathcal{O}_{f}$ peut être approché par un difféomorphisme $g$ qui est linéaire au voisinage de $p_{g}$ (Voir par exemple $[\mathrm{N}]$ ). Ce difféomorphisme $g$ n'est pas transitif puisque conjugué à un produit de rotations au voisinage de $p_{g}$. 
Pour conclure, il suffit de montrer que tout difféomorphisme de $\operatorname{Diff}_{\omega}^{1}(M)$ est isotope à un tel difféomorphisme $f$. Le closing lemma montre que chaque classe d'isotopie possède un difféomorphisme $h$ ayant un point périodique. Quitte à composer $h$ avec un difféomorphisme symplectique coincidant avec l'identité hors d'un petit voisinage de ce point périodique, on peut changer arbitrairement la différentielle de ce point périodique.

2. L'intérieur $\mathcal{S}$ de $\mathcal{G}$ est, par définition, l'ensemble des difféomorphismes symplectiques de $M$ qui sont robustement transitifs. On sait (voir [DW]) que $\mathcal{S}$ contient un ouvert dense dans l'ensemble (ouvert) $\mathcal{H}$ des difféomorphismes symplectiques partiellement hyperboliques de $M$.

Rappelons que, pour les difféomorphismes non-symplectiques, l'existence d'une décomposition dominée est nécessaire à la transitivité robuste (voir [DPU, BDP]). Dans le cadre symplectique, une telle décomposition dominée est toujours une structure d'hyperbolicité partielle (Voir le paragraphe suivant ces remarques). Il est donc naturel de demander :

Question 1. Un difféomorphisme symplectique robustement transitif est-il partiellement hyperbolique?

La réponse à cette question est positive quand $\operatorname{dim} M \leq 4$ (voir [N] pour la dimension 2 et $\left[\mathrm{A}_{2}\right]$ pour la dimension 4). Récemment, V. Horita et A. Tahzibi $[\mathrm{HT}]$ ont annoncé une réponse positive à cette question, en toute dimension.

3. Nous avons vu que, quand $\omega$ est une forme volume, l'analogue du théorème 1 est démontré dans $[\mathrm{BC}]$. En fait, de nombreux arguments de $[\mathrm{BC}]$ sont repris dans la démonstration du théorème 1 , et nous ajoutons des arguments spécifiques au cas symplectique pour expliquer ce qui se passe près des points périodiques (la difficulté provenant du fait qu'ils ne sont pas forcément hyperboliques).

4. Si de plus $M$ est exacte symplectique (ce qui signifie que la forme symplectique $\omega$ est exacte $(\omega=d \lambda)$ ), on peut s'intéresser aux difféomorphismes qui sont exacts symplectiques (ceux pour lesquels la forme $f^{*} \lambda-\lambda$ est exacte). Le théorème 1 est aussi valable dans la catégorie des difféomorphismes exacts symplectiques : en effet, les perturbations faites dans la démonstration du théorème 1 sont toutes à supports dans une réunion disjointe et finie de boules, et ne changent donc pas le caractère exact symplectique du difféomorphisme.

Pour tout difféomorphisme $f$ d'une variété compacte et pour tout compact $\Lambda$ invariant par ce difféomorphisme, $[\mathrm{BDP}]$ définit la notion de décomposition dominée la plus fine au-dessus de $\Lambda$ (on dit que cette décomposition dominée est dégénérée si elle ne contient qu'un seul terme, l'espace tangent lui-même). Dans le cas symplectique, Mañé a remarqué dans $[\mathrm{M}]$ (voir aussi $\left[\mathrm{A}_{2}\right.$, BV]) que chaque sous-fibré de cette décomposition dominée est uniformément hyperbolique (uniformément contracté ou uniformément dilaté) sauf éventuellement un sous-fibré qui est alors appelé fibré central de $\Lambda$. L'espace stable (somme des fibrés uniformément contractants) et l'espace instable sont alors de même dimension.

Si la décomposition dominée au-dessus de $M$ n'a pas de direction centrale, on dit que $f$ est un difféomorphisme d'Anosov; s'il existe une direction centrale mais que la dimension de l'espace stable (ou de l'espace instable) est non nulle, on dit que $f$ est partiellement hyperbolique.

On déduit du théorème 1 un corollaire qui permet de généraliser les résultats contenus dans $\left[\mathrm{A}_{2}\right]$ et $[\mathrm{N}]$ : 
Corollaire 1.2. Soit $(M, \omega)$ une variété compacte connexe symplectique de dimension 4. Il existe un $G_{\delta}$ dense $\mathcal{G}$ de $\operatorname{Diff}_{\omega}^{1}(M)$ tel que tout difféomorphisme $f \in \mathcal{G}$ est transitif et vérifie une et une seule des trois propriétés suivantes:

- $f$ est Anosov;

- $f$ est partiellement hyperbolique et robustement transitif. De plus, $f$ possède une suite d'orbites périodiques elliptiques ayant un espace central de dimension deux correspondant $\grave{a} E_{c}$ qui converge vers $M$ en topologie de Hausdorff.

- $f$ possède une suite d'orbites périodiques complètement elliptiques qui converge vers $M$ en topologie de Hausdorff.

Nous conjecturons que cette situation se généralise en toute dimension :

Conjecture 1.3. Soit $(M, \omega)$ une variété compacte connexe symplectique. Il existe un $G_{\delta}$ dense $\mathcal{G}$ de $\operatorname{Diff}_{\omega}^{1}(M)$ tel que tout difféomorphisme $f \in \mathcal{G}$ est transitif et vérifie l'une des trois propriétés suivantes:

- $f$ est Anosov;

- $f$ est partiellement hyperbolique et robustement transitif. De plus, $f$ possède une suite d'orbites périodiques elliptiques dont le sous-espace central est l'espace central de la structure partiellement hyperbolique qui converge vers $M$ pour la topologie de Hausdorff.

- $f$ possède une suite d'orbites périodiques complètement elliptiques qui converge vers $M$ en topologie de Hausdorff.

Le théorème 1 est une conséquence d'un résultat de perturbation que nous présentons maintenant. Pour tout $\varepsilon>0$, une $\varepsilon$-pseudo-orbite de $f$ est une suite $\left(x_{n}\right)$ de points de $M$ telle que pour tout $n$, la distance $d\left(f\left(x_{n}\right), x_{n+1}\right)$ est inférieure à $\varepsilon$.

Pour tout couple $(x, y)$ de points de $M$, on définit la relation $x \dashv y$ si pour tout $\varepsilon>0$, il existe une $\varepsilon$-pseudo-orbite $x=x_{0}, x_{1}, \ldots, x_{n}=y$ avec $n \geq 1$ qui joint $x$ à $y$.

Théorème 2. Soit $f$ un difféomorphisme symplectique d'une variété symplectique compacte $(M, \omega)$. On suppose qu'aucune orbite périodique de $f$ n'a de résonance non triviale. Alors, pour tout voisinage $\mathcal{U}$ de $f$ dans $\operatorname{Diff}_{\omega}^{1}(M)$ et tout couple $(x, y)$ de points de $M$ vérifiant $x \dashv y$, il existe un difféomorphisme $g \in \mathcal{U}$ et un entier $n \geq 1$ tel que $g^{n}(x)=y$.

Remarque 1.4. 1. La condition sur les orbites périodiques est générique dans $\operatorname{Diff}_{\omega}^{1}(M)$ (voir section 4.1).

2. Il suffit en fait qu'il n'y ait pas de résonance non triviale entre les valeurs propres de module 1.

3. Ce résultat est vrai pour d'autres espaces $\mathcal{S}$ de difféomorphismes. Nous avons dégagé deux propriétés suffisantes : la première appelée axiome de perturbation locale (déjà énoncée par $[P R])$ permet d'obtenir les closing et connecting lemmas par des perturbations dans $\mathcal{S}$; la seconde appelée contournabilité des orbites périodiques nous permet pour les pseudo-orbites joignant $x$ à $y$ d'éviter des passages trop proches des orbites périodiques de basses périodes.

4. Comme nous l'expliquerons en section 2.1, si le difféomorphisme $f$ du théorème 2 est de classe $C^{r}, r \geq 1$, alors le difféomorphisme g peut également être choisi de classe $C^{r}$.

Comme dans $[\mathrm{BC}]$, la démonstration utilise trois ingrédients : 
- les perturbations locales (section 2) : la formalisation des perturbations réalisées pour obtenir le connecting lemma a donné lieu à la définition de boîtes de perturbations. Elles sont supportées par de petits ouverts disjoints d'un nombre suffisamment grand d'itérés.

- une analyse de la dynamique près des orbites périodiques de basse période (section 4) : les orbites périodiques de basse période ne sont contenues dans aucune boîte de perturbation. Il faut donc les traiter séparément.

- une analyse de la dynamique globale (section 3), afin de recouvrir l'ensemble des orbites de la variété par des boîtes de perturbation disjointes.

Un but de cet article a été de reprendre et de formaliser (sections 2 et 3) les différents arguments de $[\mathrm{BC}]$ dans le cadre plus général des espaces de difféomorphismes.

La difficulté principale dans le cadre symplectique (hormis le fait qu'il faut faire des perturbations symplectiques, ce qui ne pose pas de problème), est que les points périodiques des difféomorphismes symplectiques peuvent être stablement elliptiques; on ne peut donc pas se ramener au cas où tous les points périodiques sont hyperboliques. C'est pourquoi en section 4 nous traitons le cas des points périodiques en détail (sauf le cas des points hyperboliques, traité dans $[\mathrm{BC}])$.

Dans le cas d'une variété non compacte ${ }^{1}$, on munit l'ensemble $\operatorname{Diff}_{\omega}^{1}(M)$ des difféomorphismes symplectiques de $M$ de la $C^{1}$-topologie forte de Whitney ([PR]). Il existe alors des exemples de difféomorphismes symplectiques qui sont robustement non transitifs (voir exemples suivants). On obtient un résultat concernant les orbites "bornées" :

Théorème 3. Soit $(M, \omega)$ une variété connexe symplectique non compacte. Il existe un $G_{\delta}$ dense $\mathcal{G}$ de $\operatorname{Diff}_{\omega}^{1}(M)$ tel que, pour tout $f \in \mathcal{G}$, l'ensemble des points de $M$ dont l'orbite positive est relativement compacte est d'intérieur vide.

Remarque 1.5. 1. L'ensemble des points dont l'orbite positive n'est pas relativement compacte est toujours un $G_{\delta}$ de $M$ (en effet, $M$ est une union croissante dénombrable de compacts $K_{n}$, et l'ensemble des points ayant un itéré positif hors de $K_{n}$ est ouvert; l'ensemble des points dont l'orbite positive n'est pas bornée est l'intersection de ces ouverts), le théorème 3 nous dit que pour un difféomorphisme générique, ce $G_{\delta}$ est dense.

2. Pour les mêmes raisons que le théorème 1, ce résultat est valable pour les difféomorphismes exacts symplectiques si on travaille sur une variété exacte symplectique.

Donnons quelques exemples de comportements des dynamiques symplectiques sur les variétés ouvertes.

Exemple 1. 1. Voici un exemple de difféomorphisme robustement transitif sur une variété ouverte : étant donné un difféomorphisme d'Anosov du tore $\mathbb{T}^{2}$, on considère sa restriction $\grave{a} \mathbb{T}^{2}$ privé d'un des points fixes. Pour la topologie de Whitney, ce difféomorphisme du tore épointé est encore structurellement stable et en particulier robustement transitif.

2. Plus généralement, pour un difféomorphisme générique d'une variété symplectique $M$ de volume fini, l'ensemble des points dont l'orbite positive est relativement compacte est dense dans $M$. En effet, le $C^{1}$-closing lemma (voir [PR], théorème 11.3) nous dit qu'il existe un $G_{\delta}$ dense $\mathcal{G}$ de $\operatorname{Diff}_{\omega}^{1}(M)$ tel que les points périodiques d'un élément de $\mathcal{G}$ forment une partie dense de $M$; dans ce cas, l'ensemble des points d'orbite positive relativement compacte est donc aussi dense dans $M$.

1. On ne considère bien sûr que des variétés paracompactes! 
3. Bien sûr, en volume infini, le comportement des difféomorphismes symplectiques génériques est très différents. Donnons un exemple d'une dynamique qui est robustement non-transitive et pour laquelle l'ensemble des points d'orbite positive relativement compacte est vide: considérons $\mathbf{R}^{2 n}$ muni de sa forme symplectique usuelle et $f$ une translation de vecteur non nul; il existe un voisinage $\mathcal{V}$ de $f$ (pour la topologie $C^{1}$ forte de Whitney) tel que l'orbite positive de tout point de $\mathbf{R}^{2 n}$ sous un élément quelconque de $\mathcal{V}$ n'est pas relativement compacte (et même mieux, sort définitivement de tout compact). Dans ce cas, pour chaque élément de $\mathcal{V}$, l'ensemble des points d'orbite relativement compacte est vide.

Question 2. Etant donnée une variété symplectique $(M, \omega)$ connexe non compacte mais de volume fini, est-il vrai qu'il existe un $G_{\delta}$ dense de $\operatorname{Diff}_{\omega}^{1}(M)$ dont tous les éléments sont transitifs?

Il est à noter que si Diff $\omega_{\omega}^{1}(M)$ est muni de la $C^{1}$-topologie forte de Whitney, les points dont l'orbite converge vers l'infini (i.e. est propre) n'ont pas de boîtes de perturbation : ceci est l'une des difficultés pour résoudre la question 2. En particulier, dans le cas non compact, le théorème 2 n'est plus vrai. On a alors besoin, pour démontrer le théorème 3, d'un énoncé adapté au cas non compact, et pour cela d'introduire une nouvelle relation.

Soit $K$ un compact invariant par $f$. On définit sur $M$ une relation $\dashv_{K}$ de la façon suivante :

Définition 1.6. Si $x$ et $y$ sont deux points de $M$, on dira que $y$ est un itéré par chaînes de $x$, à sauts dans $K$ et on notera $x \dashv_{K} y$, si pour tout $\varepsilon>0$, il existe une $\varepsilon$-pseudo-orbite $x=x_{0}, x_{1}, \ldots, x_{n}=y$ à valeurs dans $M$ avec $n \geq 1$ telle que :

$$
\forall i \in\{1, \ldots, n-2\}, f\left(x_{i}\right) \neq x_{i+1} \Rightarrow \max \left\{d\left(x_{i}, K\right), d\left(x_{i+1}, K\right)\right\}<\varepsilon .
$$

En d'autres termes, on demande que pour tout $\varepsilon>0, x$ soit joignable à $y$ par une $\varepsilon$-pseudoorbite dont tous les sauts, sauf éventuellement le premier et le dernier, sont dans un $\varepsilon$-voisinage de $K$. L'intérêt de permettre des sauts (initial et final) près de $x$ et de $y$ (et donc éventuellement loin de $K$ ) est qu'ainsi cette relation est fermée.

On peut alors donner un énoncé analogue au théorème 2 qui est valable sur les variétés non compactes :

Théorème 4. Soit $f$ un difféomorphisme symplectique d'une variété symplectique $(M, \omega)$ et soit $K$ une partie compacte de $M$ invariante par $f$. On suppose qu'aucune orbite périodique de $f$ n'a de résonance non triviale. Alors, pour tout voisinage $\mathcal{U}$ de $f$ dans $\operatorname{Diff}_{\omega}^{1}(M)$ et tout couple $(x, y)$ de points de $K$ vérifiant $x \dashv_{K} y$, il existe un difféomorphisme $g \in \mathcal{U}$ et un entier $n \geq 1$ tel que $g^{n}(x)=y$.

Ce résultat reste vrai si $x$ et $y$ sont deux points de $M$ (et non nécessairement de $K$ ) tels que $x \neq y$ et $x \dashv_{K} y$.

De plus, si $V$ est un voisinage fixé de $K \cup\left\{x, f(x), y, f^{-1}(y)\right\}$, on peut demander que $\{z \in$ $M ; g(z) \neq f(z)\}$ est contenu dans $V$.

Remerciements. Le referee a lu en profondeur cet article et a permis d'en améliorer la présentation. Nous l'en remercions. 


\section{Perturbations locales}

La clef de la démonstration des théorèmes 2 et 3 est un lemme de perturbation initialement montré par Hayashi pour créer des connections de variétés invariantes d'orbites périodiques et de ce fait appelé "connecting lemma". Le connecting lemma a été adapté au cas symplectique dans $\left[\mathrm{X}, \mathrm{A}_{1}\right]$. L'énoncé que nous donnons ici utilise la notion de boîtes de perturbations introduites dans $[\mathrm{BC}]$ et que nous rappelons en sections 2.2 et 2.3 .

\subsection{Espaces de difféomorphismes}

Étant donné un difféomorphisme $h$ d'une variété $M$, on définit son support comme l'adhérence de l'ensemble des points $x$ de $M$ tels que $h(x) \neq x$.

On appellera espace de difféomorphismes $\mathcal{S}$ d'une variété $M$ tout ensemble de difféomorphismes de $M$ muni d'une topologie.

Pour tout difféomorphisme $f$ d'un espace de difféomorphismes $\mathcal{S}$, un voisinage flexible de $f$ dans $\mathcal{S}$ est un voisinage $\mathcal{V}$ de $f$ dans $\mathcal{S}$ vérifiant : pour tous difféomorphismes à supports disjoints $h_{1}$ et $h_{2}$ on a,

$$
h_{1} \circ f \in \mathcal{V} \text { et } h_{2} \circ f \in \mathcal{V} \Longrightarrow h_{1} \circ h_{2} \circ f \in \mathcal{V} .
$$

On dira qu'un espace de difféomorphismes $\mathcal{S}$ d'une variété riemannienne $M$ vérifie l'axiome de perturbation locale si les deux propriétés suivantes sont vérifiées :

1. Pour tout compact $K$ de $M$, pour tout $f \in \mathcal{S}$ et tout voisinage $\mathcal{U}$ de $f$ dans $\mathcal{S}$, il existe des constantes $\lambda>1$ et $\delta>0$ telles que, pour tous points $p, q$ de $K$ satisfaisant $d(p, q)<\delta$, il existe un difféomorphisme $h$ de $M$ vérifiant :

- $h$ est à support dans la boule $B(p, \lambda d(p, q))$;

- $h \circ f$ appartient à $\mathcal{U}$;

$-h(p)=q$.

2. Pour tout voisinage $\mathcal{U}_{0}$ d'un difféomorphisme $f$ de $\mathcal{S}$, il existe un voisinage flexible $\mathcal{U}$ de $f$ inclus dans $\mathcal{U}_{0}$.

Lorsque l'on munit $\mathcal{S}$ de la topologie induite par la $C^{1}$-topologie $\left(C^{1}\right.$-topologie forte de Whitney dans le cas non compact), l'axiome de perturbation locale correspond au "lift axiom" de Pugh et Robinson [PR]. Ils montrent le closing lemma pour les espaces de difféomorphismes vérifiant le lift axiom mais leur preuve n'utilise en fait que l'axiome de perturbation locale ( $\mathcal{S}$ n'a pas à être muni de la $C^{1}$-topologie dès l'instant que cet axiome est vérifié).

Comme l'ont remarqué Pugh et Robinson dans [PR, section 8(a)], l'axiome de perturbation locale est vérifié en particulier par :

- l'ensemble Diff ${ }^{1}(M)$ des difféomorphismes d'une variété compacte (muni de la $C^{1}$-topologie) ou d'une variété non compacte (muni de la $C^{1}$-topologie forte de Whitney);

- l'ensemble $\operatorname{Diff}_{v}^{1}(M)$ des difféomorphismes d'une variété compacte ou non compacte préservant une forme volume $v$;

- l'ensemble $\operatorname{Diff}_{\omega}^{1}(M)$ des difféomorphismes symplectiques d'une variété symplectique compacte ou non compacte $(M, \omega)$;

- l'ensemble Diff ${ }_{0, \omega}^{1}(M)$ des difféomorphismes exacts symplectiques d'une variété exacte symplectique compacte ou non compacte $(M, \omega)$.

Dans tous les exemples ci-dessus on peut demander aux difféomorphismes d'être de classe $C^{r}$, $r \geq 1$, pourvu que la topologie considéree soit la $C^{1}$-topologie $\left(C^{1}\right.$-topologie forte de Whitney 
dans le cas non compact). En d'autre termes, les ensembles $\operatorname{Diff}^{r}(M), \operatorname{Diff}_{v}^{r}(M)$, $\operatorname{Diff}_{\omega}^{r}(M)$ et $\operatorname{Diff}_{0, \omega}^{r}(M)$, munis de la $C^{1}$-topologie, vérifient l'axiome de perturbation locale.

\subsection{Domaines quadrillés, boîtes de perturbation}

Appelons carreau de $\mathbb{R}^{d}$, tout cube compact (pour la métrique euclidienne standard) dont les côtés sont parallèles aux axes. Un domaine quadrillé $(U, \mathcal{C})$ de raison bornée par $\sigma>1$ est un ouvert borné $U$ de $\mathbb{R}^{d}$ muni d'une famille $\mathcal{C}$ de carreaux, vérifiant les propriétés suivantes :

1. $U$ est l'union des carreaux de $\mathcal{C}$;

2. deux carreaux distincts de $\mathcal{C}$ sont d'intérieurs disjoints;

3. si $C_{1}$ et $C_{2}$ sont des carreaux de $\mathcal{C}$ d'intersection non vide (on dira qu'ils sont adjacents), le rapport de leurs côtés appartient à $\left[\sigma^{-1}, \sigma\right]$;

4. toute partie compacte de $U$ rencontre un nombre fini de carreaux de $\mathcal{C}$;

5. l'union des carreaux adjacents à un carreau donné $C$ contient le cube de même centre que $C$ qui lui est homothétique de rapport $1+\frac{2}{\sigma}$.

On dit alors que $\mathcal{C}$ est un quadrillage de $U$.

Lorsque $U$ est un cube ouvert de côtés parallèles aux axes, on considèrera un quadrillage privilégié (un quadrillage "standard") $\mathcal{C}$, donné dans [BC, section 2] ; on dira alors que le domaine quadrillé $(U, \mathcal{C})$ est un cube quadrillé. La raison du quadrillage est bornée par 2 et la famille de carreaux $\mathcal{C}$ contient un carreau central dont le côté est égal au tiers du côté de $U$.

Remarque 2.1. 1. Tout ouvert borné de $\mathbb{R}^{d}$ peut être muni ${ }^{2}$ d'une famille de carreaux qui en fait un domaine quadrillé de raison bornée par 2.

2. On peut montrer que tout cube ouvert $U$ de côte parallèle aux axes possède un unique ${ }^{3}$ quadrillage de raison bornée par 2 et contenant un carreau dont le côté est le tiers du côté de $U$ : le quadrillage qui en fait un cube quadrillé.

Soit $M$ une variété de dimension $d$. Pour toute carte $\varphi: V \rightarrow \mathbb{R}^{d}$ de la variété $M$, nous appellerons domaine quadrillé de $\varphi$ l'image inverse par $\varphi$ de tout domaine quadrillé de $\mathbb{R}^{d}$ contenu dans $\varphi(V)$. On définit de même la notion de cube quadrillé de $\varphi$. Ceci défini aussi les carreaux et le quadrillage de ces cubes ou domaines quadrillés de $\varphi$.

Définition 2.2. Soit $\mathcal{S}$ un espace de difféomorphismes d'une variété $M$. On considère $f \in \mathcal{S}$ et $\mathcal{U}$ un voisinage de $f$ dans $\mathcal{S}$. Pour tout $N>0$, on appelle boîte de perturbation $B$ de longueur $N$ pour $(f, \mathcal{U})$ tout domaine quadrillé de $M$ d'adhérence disjointe de $f^{i}(\bar{B}), i \in\{1, \ldots, N\}$ et vérifiant la propriété suivante.

Pour toute suite finie $\left\{\left(x_{i}, y_{i}\right)\right\}_{i \in\{1, \ldots, \ell\}}$ de paires de points de $B$ telle que pour tout $i \in$ $\{1, \ldots, \ell\}$, les points $x_{i}$ et $y_{i}$ appartiennent à un même carreau de $B$, il existe :

2. On peut construire progressivement les carreaux de la façon suivante : on considère dans $\mathbb{R}^{d}$ le quadrillage $\mathcal{C}_{n}$ d'echelle $2^{-n}$ dont les sommets sont les points du reseau $2^{-n} \mathbb{Z}^{d}$. Soit $U$ un ouvert borné de $\mathbb{R}^{d}$. Un cube $C$ de $\mathcal{C}_{n}$ est admissible pour $U$ si $C$ et tous les cubes de $\mathcal{C}_{n}$ qui lui sont adjacents sont contenus dans $U$. Un cube de $\mathcal{C}_{n}$, admissible pour $U$, est maximal s'il n'est pas contenu dans un cube admissible de $\mathcal{C}_{n-1}$. Le quadrillage $\mathcal{C}$ de $U$ est obtenu comme l'ensemble des cubes admissibles maximaux de $U$, pour tout $n \in \mathbb{Z}$.

3. L'unicité utilise les cinq propriétés de la définition de domaine quadrillé. La propriété 5 n'est pas une conséquence des quatre précédentes. Si on l'omet, un cube ouvert $U$ de $\mathbb{R}^{d}$ peut être quadrillé de nombreuses façons pour satisfaire les propriétés 1 à 4 , même en imposant la raison égale à 2 et un carreau central de côté égal au tiers du côté de $U$. Nous remercions Luisa Paoluzzi de nous avoir construit des exemples. Nous n'avions pas relevé cette difficulté dans une version antérieure de l'article et nous remercions le referee de nous l'avoir signalée. 
- un difféomorphisme $g \in \mathcal{U}$ coïncidant avec $f$ hors de l'union $\bigcup_{t=0}^{N-1} f^{t}(B)$,

- une suite strictement croissante $n_{0}=1<n_{1}<\cdots<n_{s} \leq \ell$, tels que $g^{N}\left(x_{n_{k}}\right)=$ $f^{N}\left(y_{n_{k+1}-1}\right)$ pour tout $k \neq s$ et $g^{N}\left(x_{n_{s}}\right)=f^{N}\left(y_{\ell}\right)$.

On appelle support de la boîte de perturbation $B$ l'union $\cup_{0}^{N} f^{t}(\bar{B})$.

Soit $f$ un élément d'un espace de difféomorphisme $\mathcal{S}$ et $\mathcal{U}$ un voisinage flexible de $f$ dans $\mathcal{S}$. Soit $\mathcal{B}$ une famille finie de boîtes de perturbations de $(f, \mathcal{U})$ de supports deux à deux disjoints. On appelle pseudo-orbite à sauts dans les carreaux des boîtes de $\mathcal{B}$ toute suite $\left(x_{n}\right)$ vérifiant, pour tout $n$, ou bien $f\left(x_{n}\right)=x_{n+1}$ ou bien $x_{n}$ et $f^{-1}\left(x_{n+1}\right)$ appartiennent à un même carreau d'une boîte de $\mathcal{B}$.

La définition des boîtes de perturbation est motivée par la propriété suivante qui découle des définitions précédentes :

Proposition 2.3. Si $x=x_{0}, x_{1}, \ldots, x_{n}=y$ est une pseudo-orbite à sauts dans les carreaux de $\mathcal{B}$ qui joint deux points $x$ et $y$ hors des supports des boîtes, il existe un difféomorphisme $g \in \mathcal{U}$ et un entier $k \geq 0$ tels que $g^{k}(x)=y$.

\subsection{Existence de boîtes de perturbation : le connecting lemma}

Dans le langage de la section 2.2, le connecting lemma est un théorème d'existence de boîtes de perturbation.

Théorème 5. Soit $\mathcal{S}$ un espace de difféomorphismes d'une variété $M$. On suppose que $\mathcal{S}$ vérifie l'axiome de perturbation locale. Alors, pour tout $f \in \mathcal{S}$, tout compact $K$ invariant par $f$ et tout voisinage $\mathcal{U}$ de $f$ dans $\mathcal{S}$, il existe $N>0$ vérifiant : pour tout point $x$ de $K$, il existe une carte locale $\varphi: U_{x} \rightarrow \mathbb{R}^{d}$ en $x$ telle que tout cube quadrillé disjoint de ses $N$ premiers itérés est une boîte de perturbation d'ordre $N$ pour $(f, \mathcal{U})$.

De plus, cette propriété est encore vérifiée par les cartes proches de $\varphi$ en topologie $C^{1}$.

Une telle carte $\varphi$ est appelée carte de perturbation pour $(f, \mathcal{U})$.

La preuve de [BC, théorème 2.1] donne le théorème 5 dans le cas où $M$ est compacte en remarquant que le lemme de perturbation A.4 de $[\mathrm{BC}]$ énonce l'axiome de perturbation locale pour $\operatorname{Diff}_{\omega}^{1}(M)$. Dans le cas où $M$ est une variété non compacte, $\left[\mathrm{PR}, \mathrm{A}_{1}\right]$ construisent des cartes $\varphi$ du théorème 5 et un entier $N$, au voisinage de tout point d'un compact invariant; la preuve de $[\mathrm{BC}]$ montre que les cubes quadrillés (disjoints de leurs $N$ premiers itérés) de ces cartes sont des boîtes de perturbations. La compacité de $K$ permet de recouvrir $K$ par un nombre fini de cartes et d'obtenir de ce fait un entier $N$ uniforme.

Remarque 2.4. Fixons une constante $\sigma>1$. Le théorème reste encore valable si l'on remplace les cubes quadrillés par des domaines quadrillés de rapport borné par $\sigma$. La démonstration est identique une fois que l'on a remarqué que les carreaux d'un domaine quadrillé de rapport borné par $\sigma$ vérifient les propriétés suivantes :

- le nombre de carreaux adjacents à un carreau donné est uniformément borné par une constante qui ne dépend que de $\sigma$ et de la dimension d de la variété $M$;

- l'union des carreaux adjacents à un carreau donné $C$ contient le cube de même centre que $C$ qui lui est homothétique de rapport $1+\frac{2}{\sigma}$.

Nous n'utiliserons pas dans cet article cette notion de domaines quadrillés. 


\section{Perturbations globales}

Afin de perturber la dynamique globale, nous cherchons dans cette partie à couvrir l'espace des orbites contenues dans un compact invariant (éventuellement $M$ quand la variété est compacte) par une famille de boîtes de perturbations de supports deux à deux disjoints. Dans cette partie, nous reprenons les arguments de perturbations globales de [BC] en formalisant leurs hypothèses à l'aide de nouvelles définitions. Ceci permet de caractériser des espaces de difféomorphismes auquels s'appliquent ces résultats : les théorèmes 2 et 4 sont valables dans les espaces de difféomorphismes vérifiant l'axiome de perturbation locale pour les difféomorphismes dont les orbites périodiques sont uniformément contournables (voir le théorème 6). Bien souvent, nous ne répéterons pas les preuves qui sont identiques à celles de [BC].

\subsection{Familles de boîtes couvrantes}

Soit $\mathcal{S}$ un espace de difféomorphismes d'une variété $M, f$ un élément de $\mathcal{S}$ et $\mathcal{U}$ un voisinage de $f$ dans $\mathcal{S}$. Soit $K \subset M$ un compact invariant.

Définition 3.1. On dit qu'un ensemble fini $\mathcal{B}$ de boîtes de perturbations pour $(f, \mathcal{U})$, de supports deux à deux disjoints est couvrant pour $K$ s'il existe une famille finie $\mathcal{D}$ de compacts de $M$ chacun contenus dans l'intérieur d'un carreau d'une boîte de $\mathcal{B}$ et un entier $k>0$ tels que tout segment d'orbite de $K$ de longueur supérieure ou égale à $k$ rencontre un compact de $\mathcal{D}$.

Par compacité de $K$, pour vérifier qu'une famille $\mathcal{B}$ est couvrante pour $K$, il suffit en fait de vérifier que tout point de $K$ a un itéré dans l'intérieur d'un carreau d'une boîte de perturbation de $\mathcal{B}$. La compacité de $K$ permet de plus d'obtenir une constante $\varepsilon>0$ et une famille $\mathcal{D}^{\prime}$ de compacts obtenus en augmentant légèrement les compacts de $\mathcal{D}$, tels que toute $\varepsilon$-pseudo-orbite de $K$ de longueur supérieure ou égale à $k$ rencontre un compact de $\mathcal{D}^{\prime}$.

Si un compact $K$ possède des orbites périodiques de basse période, il ne possède en général pas de famille couvrante : en effet, ses orbites périodiques de basse période sont disjointes des boîtes de perturbation. Ceci nous amène à définir des familles couvrantes hors d'une famille $\mathcal{V}$ d'ouverts qui seront des voisinages de ces orbites périodiques.

Soit $\mathcal{V}=\left\{V_{1}, \ldots, V_{r}\right\}$ un ensemble fini d'ouverts non vides de $M$ deux à deux disjoints.

Définition 3.2. On dit qu'un ensemble fini $\mathcal{B}$ de boîtes de perturbations pour $(f, \mathcal{U})$, de supports deux à deux disjoints est couvrant pour un compact $K$ de $M$ invariant par $f$ hors de $\mathcal{V}$ s'il existe

- un entier $k>0$ et une constante $\varepsilon>0$,

- pour tout $i \in\{1, \ldots, r\}$, un ouvert $W_{i}$ inclus dans $V_{i}$ et un compact $F_{i}$ inclus dans $W_{i}$,

- une famille finie $\mathcal{D}$ de compacts de $M$, chacun contenu dans l'intérieur d'un carreau d'une boîte de $\mathcal{B}$,

- et pour tout $i \in\{1, \ldots, r\}$, deux parties $\mathcal{D}_{e, i}$ et $\mathcal{D}_{s, i}$ de $\mathcal{D}$, telles que le support des boîtes de $\mathcal{B}$ contenant ces compacts soient contenus dans $V_{i}$,

tels que l'on ait les propriétés suivantes:

1. Tout segment de $\varepsilon$-pseudo-orbite de $f$ inclus dans $K$ de longueur supérieure ou égale à $k$ rencontre un compact $F_{i}$ ou un compact de $\mathcal{D}$.

2. Pour tout $i \in\{1, \ldots, r\}$, tout segment de $\varepsilon$-pseudo-orbite de $f$ ayant son origine hors de $V_{i}$ et son extrémité dans $W_{i}$ rencontre un compact de $\mathcal{D}_{e, i}$. 
3. Pour tout $i \in\{1, \ldots, r\}$, tout segment de $\varepsilon$-pseudo-orbite de $f$ ayant son origine dans $W_{i}$ et son extrémité hors de $V_{i}$ rencontre un compact de $\mathcal{D}_{s, i}$.

4. Pour tout $i \in\{1, \ldots, r\}$, et tous compacts $D_{e} \in \mathcal{D}_{e, i}$ et $D_{s} \in \mathcal{D}_{s, i}$, il existe une pseudoorbite à sauts dans les carreaux des boîtes de $\mathcal{B}$ ayant son origine dans $D_{e}$ et son extrémité dans $D_{s}$.

Cette définition signifie que toute $\varepsilon$-pseudo-orbite de $K$ a des retours réguliers dans les compacts de la famille $\mathcal{D}$ pendant les intervalles de temps qu'elle passe hors des ouverts $V_{i}$. Pour les intervalles de temps longs entre deux retours entre deux compacts de $\mathcal{D}$, la pseudo-orbite s'approche d'un des compacts $F_{i} \subset V_{i}$. Pour cela, elle doit passer par un compact d'entrée $D_{e}$ puis par un compact de sortie $D_{s}$; l'item 4 de la définition permet de remplacer ce segment par une pseudo-orbite à sauts dans les carreaux des boîtes de $\mathcal{B}$.

Remarques 3.3. $\quad$ 1. Dans la définition précédente, si la famille $\mathcal{V}$ est vide, une famille couvrante pour $K$ hors de $\mathcal{V}$ est alors une famille couvrante pour $K$, au sens de la définition 3.1. Réciproquement, un argument de compacité (voir la remarque suivant la définition 3.1) montre que toute famille $\mathcal{B}$ couvrante pour $K$ au sens de la définition 3.1 est aussi couvrante pour $K$ hors de $\emptyset$ au sens de la définition 3.2.

2. Quitte à diminuer $\varepsilon>0$, et à augmenter légèrement les compacts des familles $\mathcal{D}, \mathcal{D}_{e, i}$ et $\mathcal{D}_{s, i}$ ainsi que les compacts $F_{i}$, l'item 1 de la définition 3.2 est encore vérifié par toute $\varepsilon$-pseudo-orbites de longueur supérieure ou égale à $k$ et possédant un point dans un $\varepsilon$ voisinage de $K$.

Si l'on sait construire des familles de boîtes de perturbation couvrantes, les théorèmes 2 et 4 découleront de la proposition suivante :

Proposition 3.4. Supposons que le voisinage $\mathcal{U}$ de $f$ est flexible. Soit $K$ une partie compacte invariante par $f$. Soit $\mathcal{B}$ une famille de boites de perturbation pour $(f, \mathcal{U})$ couvrante pour $K$ hors d'une famille $\mathcal{V}$ d'ouverts. Soient $x, y$ deux points de $K$ (ou de $M$ si $x \neq y$ ) hors des supports des boîtes de $\mathcal{B}$ et des ouverts $V_{i}$ de $\mathcal{V}$. Si $x \dashv_{K} y$, il existe alors une perturbation $g \in \mathcal{U}$ de $f$ et un entier $n \geq 1$ tel que $g^{n}(x)=y$.

Dans le cas où $M=K$ (variété compacte), la démonstration de cette proposition est l'objet des sections $4.4,4.5$ et 4.6 de $[\mathrm{BC}]$.

L'outil essentiel dans la démonstration de cette proposition (qui se démontre exactement de la même façon, que la variété soit compacte ou non) est la proposition 2.3. Pour pouvoir appliquer convenablement la proposition 2.3 , on montre :

Lemme 3.5. Supposons que le voisinage $\mathcal{U}$ de $f$ est flexible. Soit $K$ une partie compacte invariante par $f$. Soit $\mathcal{B}$ une famille de boîtes de perturbation pour $(f, \mathcal{U})$ couvrante pour $K$ hors d'une famille $\mathcal{V}$ d'ouverts. Soient $x, y$ deux points de $M$ hors des supports des boîtes de $\mathcal{B}$ et des ouverts $V_{i}$ de $\mathcal{V}$. Si $x \dashv_{K} y$, il existe alors pour tout $\varepsilon>0$ une pseudo-orbite $x=x_{0}, x_{1}, \ldots, x_{n}=y$ joignant $x$ à y telle que pour tout $i \in\{1, \ldots, n-2\}$, si $f\left(x_{i}\right) \neq x_{i+1}$, alors $x_{i}$ et $f^{-1}\left(x_{i+1}\right)$ appartiennent à un même carreau d'une boîte de $\mathcal{B}$, et telle que $d\left(f\left(x_{0}\right), x_{1}\right)<\varepsilon$ et $d\left(f\left(x_{n-1}\right), y\right)<\varepsilon$.

Si de plus $x$ et $y$ appartiennent $\grave{a} K$, on peut demander que $f\left(x_{0}\right)=x_{1}$ et $f\left(x_{n-1}\right)=y$.

En d'autres termes, le lemme 3.5 permet de ramener tous les sauts de la pseudo-orbite, sauf éventuellement le premier et le dernier, dans les carreaux des boites de $\mathcal{B}$. 
Démonstration du lemme 3.5 : Comme $\mathcal{B}$ est couvrante pour $K$ hors de $\mathcal{V}$, on peut lui associer, comme dans la définition 3.2 et la remarque 3.3 item 2 , un entier $k>0$, un $\varepsilon>0$, des ouverts $W_{i}$, des compacts $F_{i}$, une famille $\mathcal{D}$ de compacts et des parties $\mathcal{D}_{e, i}$ et $\mathcal{D}_{s, i}$ de $\mathcal{D}$.

La propriété suivante va nous permettre de regrouper les sauts des pseudo-orbites dans les carreaux des boîtes de $\mathcal{B}$.

Si $\eta>0$ est assez petit, alors pour tout $m \in\{0, \ldots, k\}$, et toute $\eta$-pseudo-orbite $z_{0}, \ldots, z_{m}$ telle que $z_{m}$ appartient à un compact $D \in \mathcal{D}$ ou $F_{i}$, le point $f^{m}\left(z_{0}\right)$ est contenu dans l'intérieur du carreau contenant $D$ ou dans l'ouvert $W_{i}$, respectivement, et de plus $d\left(f^{m}\left(z_{0}\right), z_{m}\right)<\frac{\varepsilon}{2}$. De même, si $z_{0}$ appartient à un compact $D \in \mathcal{D}$ ou $F_{i}$, le point $f^{-m}\left(z_{m}\right)$ est contenu dans l'intérieur du carreau contenant $D$ ou dans l'ouvert $W_{i}$, respectivement, et de plus $d\left(f^{-m}\left(z_{m}\right), z_{0}\right)<\frac{\varepsilon}{2}$.

Soit $x$ et $y$ tels que $x \dashv_{K} y$ et tel que $y$ n'appartienne pas à l'orbite positive de $x$. Considérons une $\eta$-pseudo-orbite $\eta$-pseudo-orbite $x=x_{0}, x_{1}, \ldots, x_{n}=y$ joignant $x$ à $y$, et dont tous les sauts, sauf éventuellement le premier (en $x$ ) et le dernier (en $y$ ) sont contenus dans le $\varepsilon$-voisinage de $K$. Comme $y$ n'est pas sur l'orbite positive de $x$, on peut supposer que $n \geq 2 k$.

Soit $i \in\{1, \ldots, n-2\}$ tel $f\left(x_{i}\right) \neq x_{i+1}$ (on considère aussi $i=0$ si $x \in K$ et $i=n-1$ si $y \in K)$. Par définition, $x_{i}$ appartient au $\varepsilon$-voisinage de $K$. Par la remarque 3.3 item 2 , il existe $j$ avec $|i-j| \leq k$ tel que $x_{j}$ appartient à un compact $F_{i}$ ou $D \in \mathcal{D}$. L'argument ci-dessus permet alors de repousser les sauts intermediaires au temps $j$ : le choix de $\eta$, montre que la nouvelle pseudo-orbite passe par l'ouvert $W_{i}$ ou le carreau contenant $D$ au temps $j$ et n'a plus de sauts entre $x_{i}$ et $x_{j}$. En répétant cet argument on peut ainsi obtenir une $\varepsilon$-pseudo-orbite $x=y_{0}, \ldots, y_{n}=y$ joignant $x$ à $y$ et dont tous les sauts, sauf éventuellement celui en $x$ et en $y$, sont contenus dans les carreaux des boîtes de $\mathcal{B}$ ou bien dans les ouverts $W_{i}$.

Pour chaque $i$, tel que la pseudo-orbite $\left(y_{j}\right)$ rencontre $W_{i}$, on considère $j_{1}$ et $j_{2}$ les premier et derniers passages de cette pseudo-orbite dans $W_{i}$. Puisque $x$ et $y$ n'appartiennent pas à $V_{i}$, les items 2 et 3 de la définition 3.2 assurent l'existence de compacts $D_{e} \in \mathcal{D}_{e, i}$ et $D_{s} \in \mathcal{D}_{s, i}$ et $j_{e} \in\left\{1, \ldots, j_{1}-1\right\}$ et $j_{s} \in\left\{j_{2}+1, \ldots, j_{n-1}\right\}$ tels que $y_{j_{e}} \in D_{e}$ et $y_{j_{s}} \in D_{s}$. On remplace alors la pseudo-orbite entre les temps $j_{e}$ et $j_{s}$ par un pseudo-orbite à sauts dans les carreaux de $\mathcal{B}$, donnée par l'item 4 de la définition 3.2.

De cette façon, on parvient à joindre $x$ à $y$ par une pseudo-orbite dont tous les sauts, sauf éventuellement celui en $x$ et en $y$, sont contenus dans les carreaux des boîtes de $\mathcal{B}$.

Démonstration de la proposition 3.4 : Soient $x$ et $y$ deux points de $M$, hors des supports des boîtes de $\mathcal{B}$ et des ouverts de la famille $\mathcal{V}$, tels que $x \dashv_{K} y$. D'après le lemme 3.5, pour tout $\varepsilon>0$, il existe une pseudo-orbite $\left(x=x_{0}, \ldots, x_{n}=y\right)$ joignant $x$ à $y$, tels que les sauts en $x$ et en $y$ soit inférieur à $\varepsilon$, et que tous les autres sauts sont dans les carreaux des boîtes de $\mathcal{B}$. Pour chaque boîte $B \in \mathcal{B}$, par définition de boîtes de perturbation, on perturber $f$ dans le support de $B$ pour obtenir une nouvelle pseudo-orbite ayant les mêmes propriétés mais n'ayant plus de sauts dans $B$. En appliquant ce procédé successivement à chacune des boîtes de $\mathcal{B}$ on obtient un segment d'orbite pour un difféomorphisme $g$ dans $\mathcal{U}$ joignant $x_{1}$ à $x_{n-1}$.

Si $x \neq y$, pour $\varepsilon$ petit, $g^{-1}\left(x_{1}\right)$ est très proche de $x$ et $x_{n-1}$ est très voisin de $g^{-1}(y)$. Comme $x \neq y$, une petite conjugaison, par un difféomorphisme à support au voisinage de $x$ et $g^{-1}(y)$ (ce support étant assez petit pour être disjoint des boîtes de $\mathcal{B}$ ) permet d'obtenir que $y$ soit un itéré positif de $x$.

Si $x=y \in K$, le lemme 3.5 permet de choisir la pseudo-orbite $\left(x=x_{0}, \ldots, x_{n}=y\right)$ de façon que $x_{1}=f(x)$ et $f\left(x_{n-1}\right)=y$. L'orbite de $x=y$ est donc périodique pour $g$. 


\subsection{Existence de familles de boîtes couvrantes, loin des orbites périodiques de basse période}

Nous expliquons à présent comment obtenir une famille de boîtes de perturbation qui est couvrante pour un compact ne contenant pas d'orbite périodiques de basse période.

Proposition 3.6. Soit $\mathcal{S}$ un espace de difféomorphismes d'une variété de dimension d, vérifiant l'axiome de perturbations locales. Soit $f$ un élément de $\mathcal{S}$ et $\mathcal{U}$ un voisinage flexible de $f$ dans $\mathcal{S}$. Il existe alors une constante $\tau_{d}$ qui ne dépend que de la dimension d avec la propriété suivante:

Soit $K$ un compact invariant et $N$ l'entier associé à $\mathcal{U}$ et $K$ par le théorème 5 . Si K ne contient pas d'orbite périodique de période inférieure à $\tau_{d} N$, il existe une famille finie $\mathcal{B}_{0}$ de boîtes de perturbations pour $(f, \mathcal{U})$ de longueur $N$ et de supports deux à deux disjoints qui est couvrante pour $K$.

Cette proposition est une conséquence de la proposition 3.7 avec $m=10 d N$ et de la proposition 3.8. La constante $\tau_{d}$ est égale à $10 d \kappa_{d}$ où $\kappa_{d}$ est la constante donnée par la proposition 3.7 suivante.

Proposition 3.7 (Existence de tours topologiques). Pour tout entier $d$, il existe une constante $\kappa_{d}>0$ telle que, pour tout difféomorphisme $f$ d'une variété $M$ de dimension $d$, pour tout $m \in \mathbb{N}$, tout $\rho>0$, et tout compact invariant $K \subset M$ ne contenant pas de point périodique de période inférieure à $\kappa_{d} m$, il existe un ouvert $U \subset M$ ayant les propriétés suivantes :

- Pour tout point $x \in K$, il existe $n \in \mathbb{N}$ tel que $f^{n}(x)$ appartient à $U$.

- Les fermés $\bar{U}, \ldots, f^{m}(\bar{U})$ sont deux à deux disjoints.

- Les composantes connexes de $\bar{U}$ sont de diamètre inférieur à $\rho$ et rencontrent $K$.

Cette proposition est un cas particulier du théorème 3.1 de [BC]. L'ouvert $U$ est appelé tour topologique pour $K$ de longueur $m$ et de diamètre $\rho$.

Proposition 3.8. Soit $\mathcal{S}$ un espace de difféomorphismes d'une variété $M$ de dimension d, vérifiant l'axiome de perturbations locales. Soit $f$ un élément de $\mathcal{S}$ et $\mathcal{U}$ un voisinage flexible de $f$ dans $\mathcal{S}$. Soit $K$ un compact de $M$ invariant par $f$ et soit $N$ l'entier associé à $\mathcal{U}$ et $K$ par le théorème 5. Soit $U$ une tour topologique pour $K$ de longueur $10 d N$ et de diamètre $\rho$ assez petit pour que toutes les composantes connexes de $\bar{U}$ et de ses $10 d N$ premiers itérés soient chacunes incluses dans une carte de perturbation de longueur $N$ de $(f, \mathcal{U})$.

Alors, il existe une famille finie $\mathcal{B}$ de boîtes de perturbation de longueur $N$ pour $(f, \mathcal{U})$, de supports deux à deux disjoints et inclus dans $\bigcup_{t=0}^{10 d N} f^{t}(U)$, qui est couvrante pour $K$.

La démonstration de cette proposition correspond à la démonstration du corollaire 4.1 de $[\mathrm{BC}]$.

Remarque 3.9. Soit $\Lambda \subset M$ un compact qui vérifie la propriété suivante : pour tout point $x \in \Lambda$ il existe un entier $i \in\{0, \ldots, N+1\}$ tel que tous les itérés $f^{i+t}(x)$ de $x$ avec $t \in\{0, \ldots, 20 d N\}$ ne rencontrent pas $\Lambda$.

Si $K$ est un compact invariant qui ne contient pas d'orbite périodique de période inférieure ou égale à $5 \tau_{d} N=50 d \kappa_{d} N$, on peut choisir la famille $\mathcal{B}_{0}$ donnée par la proposition 3.6 pour que, de plus, les adhérences des supports des boîtes de $\mathcal{B}_{0}$ soient disjointes de $\Lambda$.

Cet argument se trouve déjà dans [BC, section 6.2.2] : le lemme 6.4 de $[\mathrm{BC}]$ montre que, pour $\rho_{0}>0$ assez petit, pour tout point $x \in M$, il existe un entier $i \in\{0, \ldots, 20 d N+N+1\}$ 
tel que tous les itérés $f^{i+t}\left(B\left(x, \rho_{0}\right)\right)$ de la boule $B\left(x, \rho_{0}\right)$ avec $t \in\{0, \ldots, 20 d N\}$ ne rencontrent pas $\Lambda$.

La proposition 3.7 appliquée à une constante $\rho<\rho_{0}$ (suffisamment petite pour pouvoir appliquer la proposition 3.8) avec $m=5 \tau_{d} N$ fournit une tour topologique $U_{0}$ pour $K$ de longueur $50 d N$ de diamètre $\rho$.

Puisque $\rho$ est inférieur à $\rho_{0}$, chaque composante connexe $C$ de $U_{0}$ possède un itéré $C^{\prime}=f^{i}(C)$ avec $i \in\{0, \ldots, 20 d N+N+1\}$ tel que $f^{t}\left(C^{\prime}\right)$ soit disjoint de $\Lambda$ pour tout $t \in\{0, \ldots, 20 d N\}$. La réunion $U$ des ouverts $f\left(C^{\prime}\right)$ est une tour topologique pour $K$ de longueur $10 d N$ et de diamètre arbitrairement petit, qui a la propriété que $f^{t}(U)$ est disjoint de $\Lambda$ pour tout $t \in\{0, \ldots, 20 d N\}$. D'après la proposition 3.8 , il existe une famille finie $\mathcal{B}_{0}$ de boîtes de perturbation pour $(f, \mathcal{U})$ de longueur $N$ couvrant $K$ et de support inclus dans $\bigcup_{t=0}^{10 d N} f^{t}(U)$.

En conséquence, les adhérences des supports de ces boîtes sont disjointes de $\Lambda$.

\subsection{Perturbations au voisinage des orbites périodiques}

Soit $f$ un difféomorphisme d'un espace $\mathcal{S}$ de difféomorphismes, $\mathcal{U}$ un voisinage de $f$ dans $\mathcal{S}$, et $N$ un entier.

Définition 3.10. On dira qu'une orbite périodique $\gamma$ d'un difféomorphisme $f \in \mathcal{S}$ est contournable pour $(\mathcal{U}, N)$ si, pour tout voisinage $V_{0}$ de $\gamma$, et pour tout entier $k$, il existe $\varepsilon>0$, des voisinages ouverts $W \subset V \subset V_{0}$ et une famille $\mathcal{B}$ de boites de pertubations pour $(f, \mathcal{U})$, de longueur $N$ et de supports disjoints, vérifiant les propriétés suivantes :

1. Les supports des boîtes de $\mathcal{B}$ sont contenues dans $V$.

2. Il existe deux familles $\mathcal{D}_{e}$ et $\mathcal{D}_{s}$ de compacts contenus dans l'intérieur de carreaux de boîtes de $\mathcal{B}$ telles que toute $\varepsilon$-pseudo-orbite ayant son origine hors de $V$ (resp. dans $W$ ) et son extrémité dans $W$ (resp. hors de $V$ ) possède un point dans un compact de $D_{e}$ (resp. de $\left.D_{s}\right)$.

3. Pour tout compact $D_{e} \in \mathcal{D}_{e}$ et tout compact $D_{s} \in \mathcal{D}_{s}$ il existe une pseudo-orbite à sauts dans les carreaux des boîtes de $\mathcal{B}$, d'origine dans $D_{e}$ et d'extrémité dans $D_{s}$.

4. Pour tout point $x$ appartenant à l'adhérence d'une boîte de $\mathcal{B}$, le temps de retour du point $f^{N}(x)$ dans l'union des adhérences des supports des boîtes de $\mathcal{B}$ est supérieur à $k$.

Si une orbite périodique $\gamma$ est contournable, alors, pour toute pseudo-orbite à extrémités loin de $\gamma$ mais qui s'approche très près de $\gamma$, on peut remplacer les segments de la pseudo-orbite proches de $\gamma$ par des segments de pseudo-orbite à sauts dans les carreaux de $\mathcal{B}$ prescrits par l'item 3.

Remarque 3.11. 1. Dans la définition ci-dessus, le dernier item permet, pour tout compact invariant $K$ sans orbite périodique de basse période, de construire une famille de boîtes couvrante pour $K$, de supports disjoints des supports des boîtes de $\mathcal{B}$ (voir la remarque 3.9 en prenant pour $\Lambda$ l'union des supports des boîtes de $\mathcal{B})$.

2. Remarquons qu'un difféomorphisme de surface possédant un point fixe $P$ non-hyperbolique de type selle avec trois séparatrices stables et trois séparatrices instables (voir la figure 1) n'est en général pas contournable. 

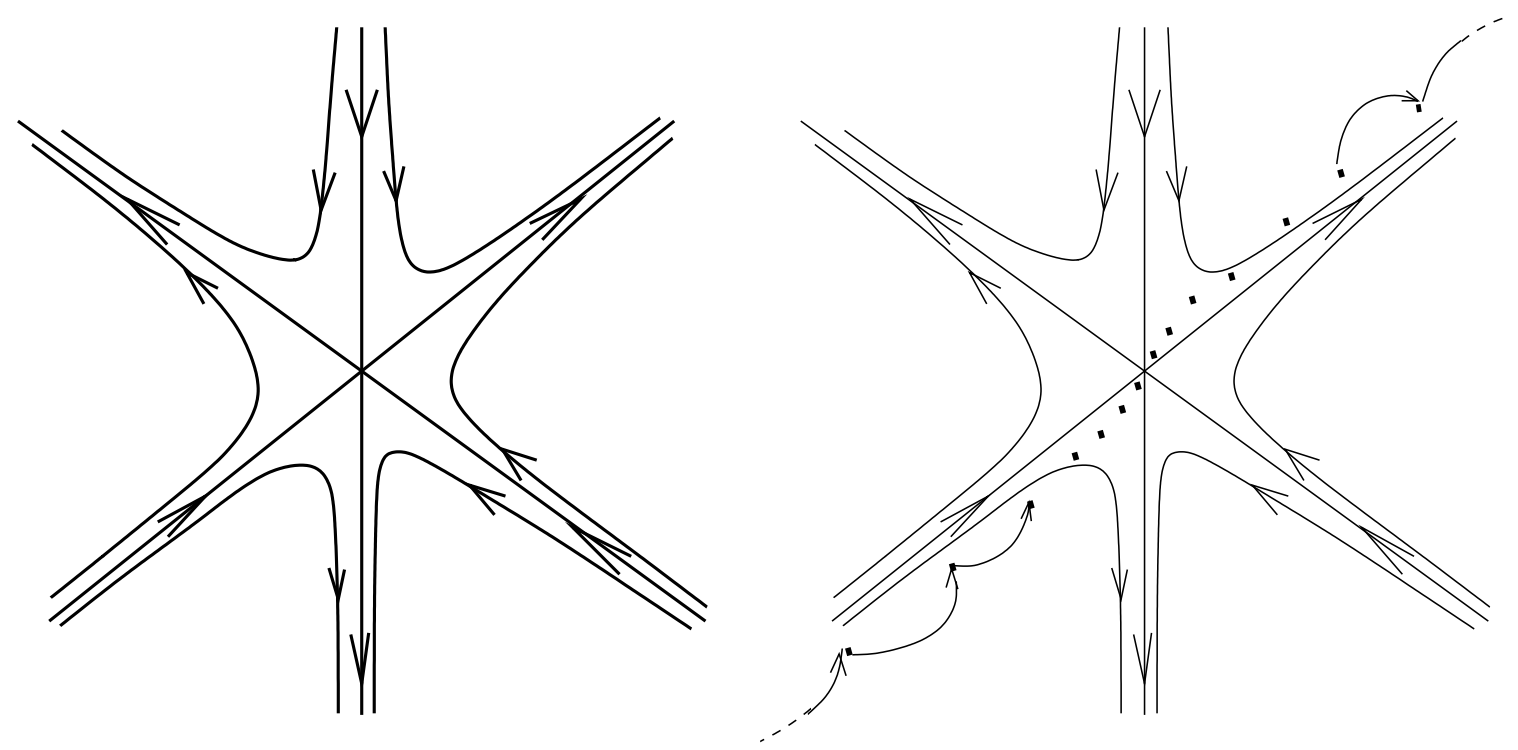

Figure 1 - Exemple de point fixe non contournable. La pseudo-orbite de la figure de droite ne peut pas être remplacée par une orbite ayant les mêmes entrée et sortie dans le voisinage du point fixe.

Soit $K$ un compact invariant de $f$. On dira que les orbites périodiques de $f$ contenues dans $K$ sont uniformément contournables si elles sont isolées ${ }^{4}$ et si, pour tout voisinage $\mathcal{U}$ de $f$, il existe un entier $N$ tel que toutes les orbites périodiques de $f$ contenues dans $K$ sont contournables $\operatorname{pour}(\mathcal{U}, N)$.

Théorème 6. Soit $\mathcal{S}$ un espace de difféomorphismes d'une variété, vérifiant l'axiome de perturbations locales. Soit $f$ un élément de $\mathcal{S}$ dont les orbites périodiques sont isolées et $K$ un compact invariant par $f$ dont les orbites périodiques sont uniformément contournables. Soient $\mathcal{U}_{0}$ un voisinage de $f$ dans $\mathcal{S}$, et $(x, y)$ un couple de points de $K$ (ou de $M$ si $x \neq y$ ) vérifiant $x \dashv_{K} y$. Alors, il existe $g \in \mathcal{U}_{0}$ tel et un entier $n>0$ tel que $g^{n}(x)=y$.

Lorsque $x \neq y$, nous pouvons éliminer à l'aide du lemme suivant le cas où l'un des points $x$ ou $y$ est périodique.

Lemme 3.12. Soit $f$ un difféomorphisme dont les orbites périodiques sont isolées ayant un compact $K$ invariant. Soient $x$ et $y$ deux points distincts tels que y n'appartient pas à l'orbite positive de $x$ et tels que $x \dashv_{K} y$.

Alors, il existe $x^{\prime}$ et $y^{\prime}$ arbitrairement proches de $x$ et $y$ respectivement tels que $x^{\prime} \dashv_{K} y^{\prime}$ et tels que ou bien $y^{\prime}$ est sur l'orbite positive de $x^{\prime}$, ou bien $x^{\prime}$ et $y^{\prime}$ ne sont pas périodiques.

Démonstration : Étant donnés des voisinages $U_{x}$ et $U_{y}$ de $x$ et $y$ respectivement, on construit par récurrence une suite de couples $\left(x_{n}, y_{n}\right)$ tels que $x_{n} \dashv_{K} y_{n}$ et des suites de boules compactes $\left(B_{x, n}\right),\left(B_{y, n}\right)$ vérifiant :

${ }_{-} B_{x, n+1} \subset B_{x, n} \subset U_{x}$ et $B_{y, n+1} \subset B_{y, n} \subset U_{y}$ pour tout $n$.

4. Une orbite périodique de période $k$ est dite isolée, si ses points sont des points fixes isolés de $f^{k}$. 
- $B_{x, n}$ est centrée en $x_{n}$ et $B_{y, n}$ centrée en $y_{n}$ pour tout $n$.

- Les rayons de $\left(B_{x, n}\right)$ et de $\left(B_{y, n}\right)$ tendent vers 0.

- $B_{x, n}$ et $B_{y, n}$ sont disjointes de leurs $n$ premiers itérés pour tout $n$.

Les points $x^{\prime}$ et $y^{\prime}$ sont alors les intersections des boules $B_{x, n}$ et des boules $B_{y, n}$ respectivement. Ils vérifient $x^{\prime} \dashv_{K} y^{\prime}$, car la relation $\dashv_{K}$ est fermée (voir le commentaire suivant la définition 1.6).

Si pour une étape de cette construction, $y_{n}$ appartient à l'orbite positive de $x_{n}$, ou bien si ni $x_{n}$, ni $y_{n}$ n'est périodique, alors la construction s'arrête puisque le couple $\left(x_{n}, y_{n}\right)$ convient. Nous pouvons donc supposer qu'à chaque étape, $y_{n}$ n'est pas sur l'orbite positive de $x_{n}$ et que $x_{n}$ ou $y_{n}$ est périodique.

On définit tout d'abord $x_{0}=x$ et $y_{0}=y$ et deux boules $B_{x, 0}$ et $B_{y, 0}$, centrées en $x_{0}$ et $y_{0}$ et contenues dans $U_{x}$ et $U_{y}$ respectivement. Lorsque $B_{x, n}$ et $B_{y, n}$ ont été définis, on construit les boules d'ordre $n+1$ de la façon suivante : puisque $x_{n} \dashv_{K} y_{n}$, il existe pour tout $k>0$ une suite de $1 / k$-pseudo-orbites joignant $x_{n}$ à $y_{n}$. Puisque $y_{n}$ n'est pas sur l'orbite positive de $x_{n}$, et si par exemple $x_{n}$ est périodique, cette suite de pseudo-orbites aura des points d'accumulation arbitrairement proches de $x_{n}$ différents de $x_{n}$. Comme $x_{n}$ est périodique et comme les orbites périodiques de $f$ sont isolées, on peut choisir un de ces points d'accumulation, $x_{n+1}$, qui est soit non périodique, soit périodique de période strictement plus grande que $n+1$ : on peut donc construire $B_{x, n+1}$ compacte centrée en $x_{n+1}$, de rayon au plus $\frac{1}{n+1}$ disjointe de ses $(n+1)$ premiers itérés. Par construction, on a encore $x_{n+1} \dashv_{K} y_{n}$. Si $y_{n}$ est périodique, on construit de la même façon $y_{n+1}$ arbitrairement proche de $y_{n}$ tel que $x_{n+1} \dashv_{K} y_{n+1}$. Sinon, on pose $y_{n+1}=y_{n}$. De toute façon, $y_{n+1}$ n'est pas périodique de période inférieure ou égale à $(n+1)$ et on peut construire $B_{y, n+1}$.

Si $y$ est sur l'orbite positive de $x$, la conclusion du théorème 6 est déjà satisfaite par le difféomorphisme $f$; cela vaut en particulier, si $x=y$ est périodique. Sinon le lemme précédent permet de supposer que $x$ et $y$ ne sont pas périodiques. Dans le cas $x \neq y$, si l'on sait montrer le théorème 6 pour un couple $\left(x^{\prime}, y^{\prime}\right)$ arbitrairement proche de $(x, y)$, une conjugaison proche de l'identité envoyant $(x, y)$ sur $\left(x^{\prime}, y^{\prime}\right)$ permet de conclure pour le couple $(x, y)$. Le théorème 6 est alors un corollaire direct de la proposition 3.4 et de la proposition suivante :

Proposition 3.13. Supposons que les hypothèses du théorème 6 sont vérifiées, que $x$ et y sont non périodiques et que y n'est pas sur l'orbite positive de $x$. Il existe alors un voisinage flexible $\mathcal{U}$ de $f$ contenu dans $\mathcal{U}_{0}$, une famille $\mathcal{V}$ d'ouverts disjoints et disjoints de $\{x, y\}$ et une famille $\mathcal{B}$ de boites de perturbations pour $(f, \mathcal{U})$, de supports disjoints et disjoints de $\{x, y\}$, qui est couvrante pour $K$ hors de $\mathcal{V}$.

Démonstration. La preuve de cette proposition est identique à celle donnée dans [BC, Section 6.2.2]. On choisit un voisinage flexible $\mathcal{U} \subset \mathcal{U}_{0}$ de $f$, par définition de l'axiome de perturbation vérifié par $\mathcal{S}$. Le théorème 5 associe à $\mathcal{U}$ et $K$ un entier $N$. Quitte à augmenter $N$, on suppose que toutes les orbites périodiques incluses dans $K$ sont contournables pour $(\mathcal{U}, N)$. Soit $\tau_{d}$ la constante donnée par la proposition 3.6.

Puisque $x$ et $y$ ne sont pas périodiques et que l'ensemble des orbites périodiques de période inférieure à $\tau_{d} N$ contenues dans $K$ est fini, il existe pour chacune de ces orbites périodiques $\gamma$ un voisinage $V_{0}(\gamma)$ de $\gamma$ ne contenant ni $x$ ni $y$ de façon à ce que ces voisinages soient deux à deux disjoints. On choisit ces voisinages suffisamment petits pour que les $20 d N$ premiers itérés positifs et négatifs de $x$ et de $y$ ne rencontrent pas les ouverts $V_{0}(\gamma)$. De plus, si $\gamma$ et $\gamma^{\prime}$ sont des orbites distinctes, les $20 d N$ premiers itérés de $V_{0}(\gamma)$ ne rencontrent pas $V_{0}\left(\gamma^{\prime}\right)$. 
Fixons $k=20 d N$. Ces orbites $\gamma$ étant contournables pour $(\mathcal{U}, N)$, on leur associe des voisinages $W(\gamma) \subset V(\gamma) \subset V_{0}(\gamma)$, une famille $\mathcal{B}(\gamma)$ de boîtes de perturbations de longueur $N$ et des familles $\mathcal{D}_{e}(\gamma), \mathcal{D}_{s}(\gamma)$ et $\mathcal{D}(\gamma)$ de compacts de sorte que, pour tout point $z$ appartenant à l'adhérence d'une boîte d'une famille $\mathcal{B}(\gamma)$, le retour de $f^{N}(z)$ à l'adhérence du support d'une boîte de $\mathcal{B}(\gamma)$ est supérieur à $k=20 d N$.

Considérons maintenant l'union $\Lambda$ des adhérences des supports des boîtes des familles $\mathcal{B}(\gamma)$ et de $\{x, y\}$. Par notre choix des ouverts $V_{0}(\gamma)$, le compact $\Lambda$ vérifie que, pour tout point $z \in \Lambda$, il existe un entier $i \in\{0, \ldots, N+1\}$ tel que tous les itérés $f^{i+t}(z)$ de $z$ avec $t \in\{0, \ldots, 20 d N\}$ ne rencontrent pas $\Lambda$.

Choisissons de plus des voisinages compacts $F(\gamma)$ de $\gamma$ inclus dans $W(\gamma)$.

Soit $\tilde{K}$ l'ensemble maximal invariant contenu dans $K \backslash \bigcup \operatorname{Int}(F(\gamma))$. La proposition 3.6 et la remarque 3.9 permettent de construire une famille de boîtes de perturbation $\mathcal{B}_{0}$ couvrant les orbites de $\tilde{K}$ et dont les supports sont disjoints de $\Lambda$.

Considérons la famille $\mathcal{B}=\mathcal{B}_{0} \cup \bigcup_{\gamma} \mathcal{B}(\gamma)$. C'est une famille de boîtes de perturbation de longueur $N$ pour $(f, \mathcal{U})$ de supports deux à deux disjoints et disjoints de $\{x, y\}$. Soit $\mathcal{V}$ la famille des ouverts $V(\gamma)$. Nous allons voir que la famille $\mathcal{B}$ est couvrante pour $K$ hors de $\mathcal{V}$.

Notons $\mathcal{D}_{0}$ la famille de compacts contenus dans l'intérieur des carreaux des boîtes de $\mathcal{B}_{0}$ donnée par la définition 3.1. Notons $\mathcal{D}$ une famille de compacts contenus dans l'intérieur des carreaux de $\mathcal{B}$ qui contient les familles $\mathcal{D}(\gamma)$ et telle que chaque compact de $\mathcal{D}_{0}$ est contenu dans l'intérieur d'un compact de $\mathcal{D}$. Les trois dernières propriétés annoncées par la définition 3.2 sont vérifiées par les ouverts $W(\gamma)$ et les familles de compacts $\mathcal{D}_{e}(\gamma)$ et $\mathcal{D}_{s}(\gamma)$ pour un certain $\varepsilon>0$. Il reste donc à vérifier la propriété 1 de cette définition.

Pour montrer cette dernière propriété, remarquons d'abord que tout segment d'orbite de $f$ suffisamment grand, inclus dans $K$ et disjoint des ouverts $\operatorname{Int}(F(\gamma))$ possède des points arbitrairement proche de $\tilde{K}$, maximal invariant hors de l'union des $\operatorname{Int}(F(\gamma))$. Tout point de $\tilde{K}$ possède un itéré (en temps uniformément borné) dans un des compacts de $\mathcal{D}_{0}$. On en déduit qu'un point suffisamment proche de $\tilde{K}$ possède un itéré en temps borné dans l'un des compacts de $\mathcal{D}$. Quitte à réduire $\varepsilon$ et à augmenter légèrement les compacts de $\mathcal{D}$, c'est encore vrai pour les $\varepsilon$-pseudo-orbites : toute $\varepsilon$-pseudo-orbite de longueur supérieure à une constante donnée et disjointe des ouverts $\operatorname{Int}(F(\gamma))$ passe dans un compact de $\mathcal{D}$.

\section{Au voisinage des points périodiques d'un difféomorphisme symplectique}

Le but de cette partie est de vérifier que les orbites périodiques d'un difféomorphisme symplectique générique d'une variété compacte sont contournables.

Théorème 7. Soit $(M, \omega)$ une variété symplectique. Il existe un $G_{\delta}$ dense $\mathcal{G}$ de $\operatorname{Diff}_{\omega}^{1}(M)$ tel que pour tout $f \in \mathcal{G}$ et tout compact $K$ invariant par $f$ les orbites périodiques de $f$ incluses dans $K$ sont uniformément contournables.

La proposition 4.2 donnera un énoncé légèrement plus précis. 


\subsection{Généralités sur les points périodiques des difféomorphismes symplec- tiques}

Considérons un difféomorphisme symplectique $f$ d'une variété $M$ de dimension $2 d$. Pour tout point périodique $p$ de période $\tau$, la différentielle $D f^{\tau}(p)$ est alors un isomorphisme symplectique de $T_{p} M$. Nous notons $\left\{\lambda_{1}, \ldots, \lambda_{2 d}\right\}$ l'ensemble de ses valeurs propres comptées avec multiplicité.

Définition 4.1. Une relation de résonance entre les valeurs propre $\left(\lambda_{i}\right)$ est une relation de la forme

$$
\lambda_{i}=\prod_{j=1}^{2 d} \lambda_{j}^{k_{j}},
$$

où $i$ appartient $\grave{a}\{1, \ldots, 2 d\}$ et où $\left(k_{1}, \ldots, k_{2 d}\right)$ est un $2 d$-uplet d'entiers naturels pour lequel ou bien $k_{i} \neq 1$, ou bien il existe $j \neq i$ tel que $k_{j} \neq 0$.

Rappelons que l'ensemble des valeurs propres d'un isomorphisme symplectique peut être ordonné pour que $\lambda_{i+d}=\lambda_{i}^{-1}$ pour tout $i \in\{1, \ldots, d\}$. Les valeurs propres de $D f(p)$ vérifient donc toujours les relations de résonance :

$$
\lambda_{i}=\lambda_{i} \prod_{k=1}^{d}\left(\lambda_{k} \lambda_{d+k}\right)^{\alpha_{k}} .
$$

Une relation de résonance autre qu'une de celles écrites ci-dessus s'appelle une résonance non triviale. C. Robinson à montré dans $[\mathrm{R}]$ qu'il n'y a génériquement pas de résonance non-triviale :

Théorème 8 (Robinson). Il existe un $G_{\delta}$ dense $\mathcal{G}_{0}$ de $\operatorname{Diff}_{\omega}^{1}(M)$ tel que, pour tout $f \in \mathcal{G}_{0}$, pour tout point périodique $p$ de $f$ de période notée $\tau$, les valeurs propres de $D f^{\tau}(p)$ ne présentent pas de résonance non triviale.

En particulier 1 n'est pas valeur propre et $p$ est isolé parmi les orbites périodiques de période $\tau$.

Nous disons que les valeurs propres de $D f^{\tau}(p)$ sont simples si elles sont toutes de multiplicité 1. Dans ce cas (puisque c'est une application linéaire symplectique) 1 n'est pas valeur propre.

Le but de cette partie est de montrer qu'un difféomorphisme $f \in \mathcal{G}_{0}$ a pour tout compact $K$ invariant par $f$ ses orbites périodiques incluses dans $K$ uniformément contournables.

Proposition 4.2. Si les valeurs propres des orbites périodiques d'un difféomorphisme symplectique $f$ ne vérifient pas de relation de résonance non triviale, pour tout compact $K$ invariant par $f$, les orbites périodiques de $f$ incluses dans $K$ sont uniformément contournables.

La preuve de la proposition 4.2 (et donc du théorème 7) est l'objet du reste de cette section.

Si $f \in \mathcal{G}_{0}$, si $p$ est un point périodique de $f$, de période notée $\tau$, les valeurs propres de $D f^{\tau}(p)$ sont donc toutes distinctes. On note $E$ le sous-espace central de $D f^{\tau}(p)$, i.e. le sousespace invariant associé aux valeurs propres de module 1, et $H$ celui qui est associé aux valeurs propres de module différent de 1 ( $E$ et $H$ sont tous deux de dimension paire). On dit alors que :

- $p$ est un point périodique hyperbolique de $f$ si $E=\{0\}$;

- $p$ est un point périodique complètement elliptique de $f$ si $H=\{0\}$;

- $p$ est un point périodique elliptique de $f$ si $E \neq\{0\}$ (ce qui contient les points complètement elliptiques); $\operatorname{dim} H$ (resp. $\operatorname{dim} E$ ) est alors sa dimension hyperbolique (resp. elliptique). 
Le cas des points périodiques hyperboliques a été étudié dans [BC] (il est rappelé en proposition 4.10) et nous ne le traiterons pas. Dans les autres cas, il est plus facile de considérer la dynamique d'une application linéaire (la différentielle de $f$ le long de l'orbite périodique). Nous nous ramenons à cette situation en section 4.2.

Plutôt que de traiter directement le cas des points périodiques elliptiques (c'est la proposition 4.17 ), nous avons choisi de commencer par les points complètement elliptiques (proposition 4.14), cas un peu plus simple et qui est une bonne introduction au cas général.

\subsection{Linéarisation du problème de contournabilité d'une orbite périodique}

Définition 4.3. Soit $L \in G L\left(\mathbb{R}^{d}\right)$ une application linéaire inversible, et soit $N_{0}$ un entier naturel. On dit que le point fixe $0=(0, \ldots, 0) \in \mathbb{R}^{d}$ est contournable pour $N_{0}$ si pour tout entier $k>0$, pour tout $C^{1}$-voisinage $\mathcal{W}$ de l'identité dans $\operatorname{Diff}^{1}\left(\mathbb{R}^{d}\right)$, il existe $\delta>0$, des voisinages ouverts bornés $W \subset V$ de 0 , une famille $\tilde{\mathcal{C}}$ de cubes quadrillés de $\mathbb{R}^{d}$ et pour tout cube $\tilde{C} \in \tilde{\mathcal{C}}$ un difféomorphisme $\varphi_{\tilde{C}} \in \mathcal{W}$, de façon que la famille $\mathcal{C}=\left\{\varphi_{\tilde{C}}(\tilde{C}), \tilde{C} \in \tilde{\mathcal{C}}\right\}$ vérifie les propriétés suivantes :

1. Pour tout cube $C \in \mathcal{C}$ les adhérences des itérés $L^{j}(C), j \in\left\{0, \ldots, N_{0}\right\}$, sont deux-à-deux disjointes. On appellera support de $C$ l'union $\bigcup_{j=0}^{N_{0}} L^{j}(C)$.

2. L'adhérences des supports des $C \in \mathcal{C}$ sont deux à deux disjoints.

3. Les supports des cubes quadrillés de $\mathcal{C}$ sont contenus dans $V$.

4. Il existe deux familles $\mathcal{D}_{e}$ et $\mathcal{D}_{s}$ de compacts contenus dans l'intérieur de carreaux de cubes de $\mathcal{C}$ telles que toute $\delta$-pseudo-orbite de $L$ ayant son origine hors de $V$ (resp. dans $W$ ) et son extrémité dans $W$ (resp. hors de $V$ ) possède un point dans un compact de $\mathcal{D}_{e}$ (resp. de $\mathcal{D}_{s}$ ).

5. Pour tout compact $D_{e} \in \mathcal{D}_{e}$ et tout compact $D_{s} \in \mathcal{D}_{s}$, il existe une pseudo-orbite de $L \grave{a}$ sauts dans l'intérieur des carreaux des cubes de $\mathcal{C}$, d'origine dans $D_{e}$ et d'extrémité dans $D_{s}$.

6. Pour tout point $x$ appartenant à l'adhérence d'un cube de $\mathcal{C}$, le temps de retour (pour L) du point $L^{N_{0}}(x)$ à l'adhérence du support d'un cube de $\mathcal{C}$ est supérieur à $k$.

Soit $f$ un difféomorphime d'un espace $\mathcal{S}$ de difféomorphismes, $\mathcal{U}$ un voisinage de $f$ dans $\mathcal{S}$, et $N$ un entier. Soit $p$ un point périodique de période $\tau$ de $f$. Le connecting lemma assure l'existence d'un $C^{1}$-ouvert $\mathcal{Z}$ de cartes locales en $p$ de façon que les cubes quadrillés de ces cartes, disjoints de leurs $N$ premiers itérés par $f$, soient des boîtes de perturbations. Considérons une carte $\psi \in \mathcal{Z}$ et notons $L \in G L\left(\mathbb{R}^{d}\right)$ la différentielle en 0 de $\psi(p) \circ f^{\tau}(p) \circ \psi^{-1}(0)$.

Notons $N_{0}$ la partie entière $\left[\frac{N}{\tau}\right]$.

Le but de ce paragraphe est de montrer la proposition suivante :

Proposition 4.4. Si 0 est contournable pour $L, N_{0}$ alors $p$ est contournable pour $f, \mathcal{U}, N$.

La démonstration repose sur les faits élémentaires suivants : si $p$ est un point fixe de $f$ et si $\psi$ est une carte au voisinage de $p$ telle que $\psi(p)=0$, alors, les applications $x \mapsto \lambda^{-1} \psi \circ f \circ$ $\psi^{-1}(\lambda x)$ convergent vers $L$ uniformément sur tout compact lorsque $\lambda$ tend vers 0 . Ceci permet de transporter les pseudo-orbites de $f$ (au voisinage de $p$ ) en des pseudo-orbites de l'application linéaire $L$ (et vice versa). D'autre part, le choix de la carte $\psi \in \mathcal{Z}$ assure qu'un cube quadrillé de $\mathbb{R}^{d}$ correspond à une boîte de perturbation de $f$. 
Lemme 4.5. Il existe un $C^{1}$-voisinage $\mathcal{W}$ de l'identité de $\mathbb{R}^{d}$ ayant la propriété suivante.

Soit $\varphi \in \mathcal{W}$ un difféomorphisme et $C$ un cube quadrillé de $\mathbb{R}^{d}$, tel que tous les itérés $L^{i}(\varphi(C))$, $i \in\left\{0, \ldots, N_{0}\right\}$, sont d'adhérences deux à deux disjointes. Il existe alors un nombre $\lambda_{0}>0$ tel que, pour tout $\left.\lambda \in] 0, \lambda_{0}\right]$, le cube $B=\psi^{-1}(\lambda \varphi(C))$ est une boite de perturbation pour $(f, \mathcal{U}, N)$.

Démonstration : Rapellons que, pour la topologie forte de Whitney, si le voisinage $\mathcal{W}$ de $I d$ est assez petit, la dérivée de tout $\varphi \in \mathcal{W}$ est uniformément proche de l'identité. Si $\lambda$ est assez petit, $\lambda \varphi(C)$ est dans l'image de la carte $\psi$. Ainsi pour $\mathcal{W}$ choisi assez petit, pour tout $\varphi \in \mathcal{W}$, pour tout cube quadrillé $C$ de $\mathbb{R}^{d}$ et tout $\lambda$ assez petit, $B=\psi^{-1}(\lambda \varphi(C))$ est un cube quadrillé d'une carte $C^{1}$-proche de $\psi$, au voisinage de $p$; cette carte est donc dans $\mathcal{Z}$.

Il suffit alors de remarquer que, pour $\lambda$ assez petit, les $N$ premiers itérés de $B$ restent arbitrairement voisins de l'orbite de $p$, et donc deux itérés $f^{i}(B)$ et $f^{j}(B), i, j \in\{0, \ldots, N\}$ ne peuvent avoir une intersection non-vide que si $j-i$ est un multiple de la période $\tau$. Il suffit donc de montrer que les itérés $f^{i \tau}(B), i \in\left\{0, \ldots, N_{0}\right\}$ sont deux à deux disjoints. Pour conclure, il reste à observer que $x \mapsto \lambda^{-1} \psi \circ f^{\tau} \circ \psi^{-1}(\lambda x)$ converge sur les compacts de $\mathbb{R}^{d}$ en topologie $C^{1}$ vers l'application linéaire $L$ : les $N$ premiers itérés de $B$ sont donc d'adhérences deux à deux disjointes.

Nous pouvons à présent montrer la proposition 4.4 :

Démonstration de la proposition 4.4 : Soient $V_{0}$ un voisinage de l'orbite de $p$ (réunion des $\tau$ premières images d'un voisinage de $p$ inclus dans le domaine de la carte $\psi$ ) et $k \geq 0$ un entier. On choisit un voisinage $\mathcal{W}$ de l'identité de $\mathbb{R}^{d}$ donné par le lemme 4.5.

Puisque 0 est contournable pour $L, N_{0}$, on associe à $k$ et $\mathcal{W}$ une constante $\delta$ des voisinages ouverts bornés $W^{L} \subset V^{L}$ de 0 , et une famille $\mathcal{C}^{L}=\left\{\varphi_{\tilde{C}}(\tilde{C}), \tilde{C} \in \tilde{\mathcal{C}}\right\}$, comme dans la définition 4.3 et deux familles de compacts $\mathcal{D}_{e}^{L}$ et $\mathcal{D}_{s}^{L}$ inclus dans l'intérieur de carreaux de cubes de $\mathcal{C}^{L}$.

Pour tous compacts $D_{e}^{L} \in \mathcal{D}_{e}^{L}$ et $D_{s}^{L} \in \mathcal{D}_{s}^{L}$, on considère une pseudo-orbite $o\left(D_{e}^{L}, D_{s}^{L}\right)$ de $L$, à saut dans les carreaux des cubes de $\mathcal{C}^{L}$, joignant $D_{e}^{L}$ à $D_{s}^{L}$. Remarquons que ces pseudo-orbites sont en nombre fini et l'union de ces pseudo-orbites est donc un ensemble fini, contenu dans un compact de $\mathbb{R}^{d}$.

D'après le lemme 4.5 , pour tout $\lambda$ assez petit, les cubes $\psi^{-1}(\lambda C)$, avec $C \in \mathcal{C}^{L}$ sont des boîtes de perturbation de $f, \mathcal{U}, N$. Ces boîtes de perturbations sont de supports deux à deux disjoints (voir la fin de la preuve du lemme 4.5). On note $\mathcal{B}$ la famille de ces boîtes (en oubliant la dépendance en $\lambda$ qui sera choisi ultérieurement).

Par le même argument et par la propriété 6 de la définition 4.3, si $\lambda$ a été choisi suffisamment petit, pour tout point $x$ appartenant à l'adhérence d'une boîte de $\mathcal{B}$, le temps de retour du point $f^{N}(x)$ à l'adhérence du support d'une boîte de $\mathcal{B}$ est supérieur à $k$.

On pose $W=\bigcup_{i=0}^{\tau-1}\left(\psi^{-1}\left(\lambda W^{L}\right)\right)$ et $V=\bigcup_{i=0}^{\tau-1}\left(\psi^{-1}\left(\lambda V^{L}\right)\right)$ : pour $\lambda$ assez petit, ce sont des voisinages ouverts de l'orbite de $p$ inclus dans $V_{0}$. Par construction, les adhérence des supports des boîtes de $\mathcal{B}$ sont contenues dans $V$.

On note $\mathcal{D}_{e}$ et $\mathcal{D}_{s}$ l'ensemble des compacts $D_{e}=\psi^{-1}\left(\lambda D_{e}^{L}\right)$ et $D_{s}=\psi^{-1}\left(\lambda D_{s}^{L}\right)$ respectivement. Ce sont compacts inclus dans l'intérieur de carreaux de cubes de $\mathcal{B}$.

Pour $\lambda$ assez petit, pour toute pseudo-orbite $o\left(D_{e}^{L}, D_{s}^{L}\right)$ joignant $D_{e}^{L}$ à $D_{s}^{L}$ et à sauts dans les carreaux des cubes de $\mathcal{C}^{L}$, introduite ci-dessus, l'image $\psi^{-1}\left(\lambda . o\left(D_{e}^{L}, D_{s}^{L}\right)\right)$ est bien défini, et contenu dans un voisinage arbitrairement petit de l'orbite de $p$. Puisque $f$ et $L$ sont très proches (au voisinage de l'orbite de $p$ ), il existe une pseudo-orbite de $f$ (voisine de $\psi^{-1}\left(\lambda . o\left(D_{e}^{L}, D_{s}^{L}\right)\right.$ )) à saut dans les carreaux des cubes de $\mathcal{B}$ et joignant $D_{e}$ à $D_{s}$. 
Fixons $U$ un compact de $\mathbb{R}^{d}$ qui contient dans son intérieur l'union des adhérences de $V^{L}$, $L\left(V^{L}\right)$ et $L^{-1}\left(V^{L}\right)$. Pour $\lambda$ assez petit, toute orbite contenue dans $U$ de l'application $x \mapsto$ $\lambda^{-1} \psi \circ f^{\tau} \circ \psi^{-1}(\lambda x)$ est une $\frac{\delta}{2}$-pseudo-orbite de $L$.

À ce stade, la constante $\lambda$ est fixée. On choisit alors $\varepsilon>0$ assez petit pour avoir la propriété suivante. Soit $\left(x_{i}\right)$ une $\varepsilon$-pseudo-orbite de $f$ et considérons la sous suite $\left(y_{j}=x_{j . \tau}\right)$. On suppose que les $y_{j}$ appartiennent à $\psi^{-1}(U)$. Alors la suite $\left(\lambda^{-1} \psi\left(y_{j}\right)\right)$ est une $\delta$-pseudo-orbite de $L$. Si $\varepsilon$ est choisi assez petit, toute $\varepsilon$-pseudo-orbite de $f$, joignant un point de $W$ (resp.du complémentaire de $V$ ) au complémentaire de $V$ (resp. à $W$ ) rencontre donc un compact $D_{s}$ (resp. $D_{e}$ ). L'orbite de $p$ est donc contournable pour $\mathcal{U}, N$.

\subsection{Familles couvrantes de cubes quadrillés}

Nous avons choisi dans cet article de ne travailler qu'avec des boîtes de perturbations qui sont des cubes quadrillés (voir la remarque 2.4) obtenus par homothétie-translation du cube ]-1,1[. La proposition 3.6 construit des boites de perturbations dont la forme n'est pas prescrite. De ce fait, nous devons dans cette section adapter la proposition 3.6 afin d'obtenir les cubes quadrillés de la définition 4.3.

Définition 4.6. Soient $f: \mathbb{R}^{d} \rightarrow \mathbb{R}^{d}$ un difféomorphisme, $K \subset \mathbb{R}^{d}$ un compact $f$-invariant, $N_{0} \geq 0$ un entier et $\mathcal{W}$ un voisinage de l'identité de $\mathbb{R}^{d}$ pour la topologie forte de Whitney.

1. On appellera cube quadrillé pour $\mathcal{W}$ l'image par un élément de $\mathcal{W}$ d'un cube quadrillé de $\mathbb{R}^{d}$.

2. On appellera cube quadrillé pour $\left(f, \mathcal{W}, N_{0}\right)$ tout cube quadrillé $C$ pour $\mathcal{W}$ dont les $N_{0}$ premiers itérés par $f$ sont d'adhérences deux à deux disjointes. On appellera support de $C$ l'union $\bigcup_{i=0}^{N_{0}} f^{i}(C)$.

3. On dit qu'un ensemble fini $\mathcal{C}$ de cubes quadrillés pour $\left(f, \mathcal{W}, N_{0}\right)$, dont les adhérences des supports sont deux à deux disjointes, est couvrant pour $K$ s'il existe une famille finie $\mathcal{D}$ de compacts de $\mathbb{R}^{d}$ chacun contenus dans l'intérieur d'un carreau d'un cube de $\mathcal{C}$ et un entier $k>0$ tels que tout segment d'orbite de $K$ de longueur supérieure ou égale à $k$ rencontre un compact de $\mathcal{D}$.

De même que dans la définition 3.1, par compacité de $K$, pour vérifier qu'une famille $\mathcal{C}$ est couvrante pour $K$, il suffit de vérifier que tout point de $K$ a un itéré dans l'intérieur d'un carreau d'un cube de $\mathcal{B}$.

Voici l'analogue de la proposition 3.6

Proposition 4.7. Il existe une constante $\tau_{d}$ avec la propriété suivante :

Soit $L \in G L\left(\mathbb{R}^{d}\right)$ une application linéaire inversible et $\mathcal{W}$ un $C^{1}$-voisinage de l'identité de $\mathbb{R}^{d}$ pour la topologie forte de Whitney. Pour tout entier $N_{0}$, tout compact invariant $K$ ne contenant pas d'orbite périodique de période inférieure à $\tau_{d} N_{0}$ et tout voisinage $U$ de $K$, il existe une famille finie $\mathcal{C}$ de cubes quadrillés de $\left(L, \mathcal{W}, N_{0}\right)$, dont les supports sont d'adhérences deux à deux disjointes, qui est couvrante pour $K$.

De plus les diamètres des cubes de $\mathcal{C}$ peuvent être choisis arbitrairement petits.

La proposition 3.6 était une conséquence directe des propositions 3.7 et 3.8. La proposition 3.7, qui construit des tours topologiques, s'applique sans aucun changement au contexte de la proposition 4.7. Par contre la proposition 3.8 doit être adaptée. 
Proposition 4.8. On considère un difféomorphisme $f$ de $\mathbb{R}^{d}$, un compact $K \subset \mathbb{R}^{d}$ invariant par $f$ un entier $N_{0} \geq 0$ et un voisinage de de l'identité de $\mathbb{R}^{d}$ pour la $C^{1}$-topologie forte de Whitney.

Soit $U$ une tour topologique pour $K$ de longueur $10 d N_{0}$.

Alors, il existe une famille finie $\mathcal{C}$ de cubes quadrillés pour $\left(f, \mathcal{W}, N_{0}\right)$, dont les adhérences des supports sont deux à deux disjointes, qui est couvrante pour $K$.

Idée de la démonstration : La démonstration de cette proposition correspond à la démonstration du corollaire 4.1 de $[\mathrm{BC}]$ : on pave les ouverts $U, \ldots, f^{2 i N_{0}}(U), \ldots, f^{(5 d-1) 2 N_{0}}(U)$ par des cubes quadrillés pour $\mathcal{W}$. Considérons la famille $\mathcal{C}_{0}$ de cubes de $U$ qui est l'union sur $i$ des images par $f^{-2 i N_{0}}$ des cubes de $f^{2 i N_{0}}(U)$. Le fait que $\mathcal{W}$ est ouvert permet de perturber ces cubes, de façon que leur bords soient en position générale. Un point de $U$ ne peut alors appartenir qu'à au plus $d$ bord de cubes de $\mathcal{C}_{0}$, et appartient de ce fait à au moins $4 d$ intérieurs de cubes de $\mathcal{C}_{0}$. Le même argument permet alors de mettre en position générale les bords des carreaux des cubes de $\mathcal{C}_{0}$; notons $\mathcal{C}_{1}$ cette nouvelle famille de cubes. Un point de $U$ appartient à au plus $d$ bords de carreaux des cubes de $\mathcal{C}_{1}$ : il appartient donc à au moins $3 d$ intérieurs de carreaux. On conclut en remarquant que chaque cube de la famille $\mathcal{C}_{1}$ est l'image par une application $f^{-2 i N_{0}}$ d'un cube quadrillé pour $\left(f, \mathcal{W}, N_{0}\right)$ contenu dans $f^{2 i N_{0}}(U)$.

Remarque 4.9. Cette démonstration montre le résultat plus général suivant: Soit $f$ un difféomorphisme de $\mathbb{R}^{d}$, soit $N_{0}>0$ un entier soit $K \subset \mathbb{R}^{d}$ un compact et $U \subset \mathbb{R}^{d}$ un ouvert disjoint de ses $10 d N_{0}$ premiers itérés tel que l'orbite d'un point de $K$ rencontre $U$. Finalement soit $\mathcal{W}$ un $C^{1}$-voisinage de l'identité de $\mathbb{R}^{d}$. Alors il existe une famille finie $\mathcal{C}$ de cubes quadrillés pour $\left(f, \mathcal{W}, N_{0}\right)$, dont les adhérences des supports sont deux à deux disjointes, et une famille finie $\mathcal{D}$ de compacts contenus dans les intérieurs des carreaux des cubes de $\mathcal{C}$ tels que toute orbite de $K$ rencontre un compact de $\mathcal{D}$.

Par construction, chaque cube de $\mathcal{C}$ est contenu dans un des $10 d N_{0}$ premiers itéré de $U$.

\subsection{Points périodiques hyperboliques}

La proposition suivante est contenue (au moins en substance) dans [BC, proposition 4.2] :

Proposition 4.10. Soit $\mathcal{S}$ un espace de difféomorphismes d'une variété riemannienne $M$ qui vérifie l'axiome de perturbation locale. Pour tout élément $f$ de $\mathcal{S}$, pour tout voisinage $\mathcal{U}$ flexible de $f$ dans $\mathcal{S}$ et tout compact $K$ invariant par $f$, on considère l'entier $N$ associé à $\mathcal{U}$ et $K$ par le théorème 5. Alors, toute orbite périodique hyperbolique de $f$ est contournable pour $(\mathcal{U}, N)$.

Remarque 4.11. Les orbites périodiques hyperboliques vérifient une propriété un peu plus forte que la définition 3.10. En effet, l'item 3 de la définition 3.10 devient : pour tout compact $D_{e} \in \mathcal{D}_{e}$ et tout compact $D_{s} \in \mathcal{D}_{s}$ il existe une vrai orbite d'origine dans $D_{e}$ et d'extrémité dans $D_{s}$.

La démonstration est presque identique à [BC, proposition 4.2], l'idée étant de recouvrir un domaine fondamental des variétés stables et instables par des boîtes de perturbation. La seule différence avec $[\mathrm{BC}]$ est de s'assurer que les boîtes ainsi construites vérifient la définition 3.10.4., i.e. que les adhérences des boîtes et leurs $N+k$ premiers itérés forment un ensemble de compacts deux à deux disjoints. On peut aussi considérer le cas hyperbolique comme un cas particulier du cas général elliptique traité à la section 4.6.

Il suffit pour cela de reprendre la démonstration de $[\mathrm{BC}]$ en traitant les boîtes de perturbation comme des boîtes de longueur $N+k$ au lieu de $N$. 
Remarque 4.12. La démonstration de cette proposition montre aussi que pour toute application $L \in G L\left(\mathbb{R}^{d}\right)$ hyperbolique et tout entier $N_{0}$, le point fixe 0 est contournable pour $N_{0}$.

\subsection{Points périodiques complètement elliptiques}

Proposition 4.13. Soit $L \in G L\left(\mathbb{R}^{2 d}\right)$ une application linéaire inversible de $\mathbb{R}^{2 d}$ préservant une forme linéaire symplectique $\omega$ de $\mathbb{R}^{2 d}$. Si L est complètement elliptique et n'a pas de résonnance non triviale, alors, le point fixe $0 \in \mathbb{R}^{2 d}$ est contournable pour tout entier $N_{0} \geq 1$.

Les propositions 4.4 et 4.13 impliquent donc la proposition suivante.

Proposition 4.14. Soit $(M, \omega)$ une variété symplectique. Pour tout élément $f$ de $\operatorname{Diff}_{\omega}^{1}(M)$, tout voisinage flexible $\mathcal{U}$ de $f$ dans $\operatorname{Diff}_{\omega}^{1}(M)$ et tout compact $K$ invariant, on considère l'entier $N$ associé à $\mathcal{U}, K$ par le théorème 5 . Alors, chaque orbite périodique de $f$, complètement elliptique, sans résonance non triviale et contenue dans $K$, est contournable pour $(\mathcal{U}, N)$.

Remarque 4.15. La démonstration de la proposition 4.13 montre aussi que les orbites périodiques complètement elliptiques vérifient la définition 3.10 avec $\mathcal{D}_{e}=\mathcal{D}_{s}$.

Démonstration de la proposition 4.13 : Il existe une transformation linéaire inversible $A$ telle que l'application $\tilde{L}=A^{-1}$.L.A soit sous forme canonique de Jordan réelle. Comme toutes les valeurs propres sont complexes (non-réelles) de module 1 et de multiplicité 1 , l'application $\tilde{L}$ préserve donc, pour tout $r>0$, la sphère

$$
S_{r}=\left\{\left(x_{i}\right) \in \mathbb{R}^{2 d} \mid \sum_{i=1}^{2 d} x_{i}^{2}=r^{2}\right\} .
$$

L'ensemble $K_{r}=A\left(S_{r}\right)$ est donc un ellipsoïde invariant par $L$ et son complémentaire a deux composantes connexes. On choisit une boule ouverte $V$ contenant $K_{r}$ et une boule ouverte $W$ (centrée en 0) dont l'adhérence ne rencontre pas $K_{r}$. Donc $W$ est contenu dans l'ellipsoïde plein bordé par $K_{r}$ et l'adhérence de $W$ est contenue dans $V$. De plus, $K_{r}$ sépare $W$ du complémentaire de $V$.

Puisque $L$ n'a pas de résonnance, non-triviale, la dynamique des restriction de $\tilde{L}$ sur $S_{r}$ et de $L$ sur $K_{r}$ sont transitives par chaînes ${ }^{5}$, et ne possède aucune orbite périodique.

Fixons un voisinage $\mathcal{W}$ de l'identité de $\mathbb{R}^{2 d}$, un entier $N_{0}$ et un entier $k>0$. La proposition 4.7 asssure l'existence d'une famille finie $\mathcal{C}$ de cubes quadrillés pour $\left(L, \mathcal{W}, N_{0}+k\right)$ dont les adhérences des supports sont deux à deux disjointes, et qui est couvrante pour $K_{r}$. Si les cubes de cette familles sont de diamètres assez petits, leurs supports sont contenus dans $V$. On considère désormais ces cubes comme étant des cubes quadrillés de $\left(L, \mathcal{W}, N_{0}\right)$ (les supports seront donc l'union des $N_{0}$ premiers itérés) ; cependant, comme ils proviennent de cubes quadrillés pour $\left(L, \mathcal{W}, N_{0}+k\right)$ le temps de retour dans l'adhérence du support d'un cube de $\mathcal{C}$ sera supérieur à $k$. Les cubes de $\mathcal{C}$ vérifie donc les items 1,2, 3 et 6 de la définition 4.3.

Puisque la famille $\mathcal{C}$ est couvrante pour $K_{r}$, il existe $m>0$ et une famille finie $\mathcal{D}$ de compacts contenus dans des intérieurs de carreaux de cubes de $\mathcal{C}$ telle que toute orbite de $K_{r}$ de longueur supérieure à $m$ rencontre un des compacts de $\mathcal{D}$. Quitte à augmenter légèrement les

5. On dit qu'un ensemble compact invariant par un difféomorphisme $f$ est transitif par chaînes si, pour toute $\varepsilon>0$, deux points quelconques de $K$ peuvent être joints par une $\varepsilon$-pseudo-orbite contenue dans $K$. 
compacts de $\mathcal{D}$, il existe $\delta>0$ tel que toute $\delta$-pseudo-orbite de longueur supérieure à $m$ et possédant un point $\delta$-proche de $K_{r}$ rencontre l'un de ces compacts. Puisque $K_{r}$ sépare $W$ du complémentaire de $V$, quitte à diminuer $\delta$, toute $\delta$-pseudo-orbite joignant $W$ au complémentaire de $V$ (ou réciproquement) est de longueur supérieure à $m$ et rencontre le $\delta$ voisinage de $K_{r}$. En conséquence, une telle pseudo-orbite rencontre l'un des compacts de $\mathcal{D}$. L'item 4 de la définition 4.3 est donc vérifié pour $\mathcal{D}_{e}=\mathcal{D}_{s}=\mathcal{D}$.

Finalement, considérons deux compacts $D_{e}$ et $D_{s}$ de $\mathcal{D}$. Il existe des pseudo-orbites de $K_{r}$, de sauts arbitrairement petits, et joignant un point de $D_{e}$ à un point de $D_{s}$. Le temps que cette pseudo-orbite passe entre deux retours dans les compacts de $\mathcal{D}$ est inférieur à $m$. On regroupe alors les sauts de cette pseudo-orbite lors de ces passages dans les compacts $\mathcal{D}$ (c'est à dire que si $x_{i}$ et $x_{j}$ sont deux points de cette pseudo-orbites correspondant à des passages successifs dans des carreaux de $\mathcal{D}$, on remplace les points $x_{k}, i<k<j$, par $\left.f^{k-j}\left(x_{j}\right)\right)$. Si les sauts de la pseudo-orbite initiale ont été choisis suffisamment petits, les sauts de la nouvelle pseudo-orbite seront dans l'intérieur des carreaux des cubes de $\mathcal{C}$. L'item 5 de la définition 4.3 est donc vérifié, ce qui conclut la preuve de la proposition.

\subsection{Points périodiques elliptiques (cas général)}

Proposition 4.16. Soit $L \in G L\left(\mathbb{R}^{2 d}\right)$ une application linéaire inversible de $\mathbb{R}^{2 d}$ préservant une forme linéaire symplectique $\omega$ de $\mathbb{R}^{2 d}$. Si $L$ n'a pas de résonnance non triviale, alors, le point fixe $0 \in \mathbb{R}^{2 d}$ est contournable pour tout entier $N_{0} \geq 1$.

Les propositions 4.4 et 4.16 impliquent donc la proposition suivante.

Proposition 4.17. Soit $(M, \omega)$ une variété symplectique. Pour tout élément $f$ de $\operatorname{Diff}_{\omega}^{1}(M)$, tout voisinage flexible $\mathcal{U}$ de $f$ dans $\operatorname{Diff}_{\omega}^{1}(M)$ et tout compact $K$ invariant, on considère l'entier $N$ associé à $\mathcal{U}, K$ par le théorème 5 . Alors, chaque orbite périodique de $f$ sans résonance non triviale et contenue dans $K$, est contournable pour $(\mathcal{U}, N)$.

La démonstration de la proposition 4.16 est longue et technique et fait l'objet du reste de cette section. Nous en expliquons maintenant les principales étapes :

1. On commence par travailler indépendamment dans les espaces stable $E^{s}$, instable $E^{u}$ et central $E^{c}$. Les boîtes que l'on construit alors sont de dimension $2 d$. De ce fait, une orbite qui visite 0 tout en restant proche de l'un de ces espaces, doit rencontrer les carreaux des boîtes correspondantes.

- L'application $L$ laisse invariant un ellipsoïde de $E^{c}$. On peut donc appliquer les arguments de la section 4.5 et construire des familles de cubes quadrillés et de carreaux, que nous noterons $\mathcal{C}^{c}$ et $\mathcal{D}^{c}$.

- Dans l'espace $E^{s}$, on couvre un domaine fondamental par une famille de carreaux $\mathcal{D}^{s}$ associés à des boîtes d'une famille $\mathcal{C}^{s}$. On construit de la même façon des familles $\mathcal{C}^{u}$ et $\mathcal{D}^{u}$ pour l'espace $E^{u}$.

2. On introduit ensuite les familles homothétiques $\mathcal{C}_{r}^{s}, \mathcal{C}_{r}^{u}$ et $\mathcal{C}_{r}^{c}$ images des familles $\mathcal{C}^{s}, \mathcal{C}^{u}$ et $\mathcal{C}^{c}$ respectivement par l'homothétie de rapport $r$. La proposition 4.18 annonce l'existence de constantes $r, t \in] 0,1\left[\right.$ telles que les familles $\mathcal{C}^{s}, \mathcal{C}_{r}^{u}$ et $\mathcal{C}_{t}^{c}$ permettent la contournabilité de 0 pour $L$.

- Le choix de $r$ est simple, il permet de disjoindre les supports de boîtes de $\mathcal{C}^{s}$ et de $\mathcal{C}_{r}^{u}$. 
- Le choix de $t$ est plus délicat. Une orbite qui visite un voisinage de 0 sufisamment petit sans être passée par un voisinage de $\mathcal{C}^{s}$ doit rester au voisinage de 0 dans un voisinage tubulaire étroit autour de l'espace $E^{c}$. De même, pour les orbites visitant 0 dans le passé. (C'est le lemme 4.21.) Ceci permet de choisir $t$ pour que les boîtes de $\mathcal{C}_{t}^{c}$ soient suffisamment proches de 0 , de sorte que toute orbite qui visite un petit voisinage de 0 rencontre dans le passé (un carreau d') une boîte de $\mathcal{C}^{s} \cup \mathcal{C}_{t}^{c}$ et dans le futur (un carreau d') une boîte de $\mathcal{C}_{t}^{c} \cup \mathcal{C}_{r}^{s}$.

3. En choisissant $\delta$ assez petit, les $\delta$-pseudo-orbites qui visitent 0 auront la même propriété (lemmes 4.21 et 4.22 ).

4. Finalement, on vérifie que les carreaux d'entrée peuvent être joints au carreaux de sortie par des pseudo-orbites à sauts dans les carreaux de ces familles de boîtes.

\section{Démonstration de la proposition 4.16 :}

Fixons un voisinage $\mathcal{W}$ de l'identité de $\mathbb{R}^{2 d}$ et des entiers $N_{0}$ et $k>0$. Quitte à réduire $\mathcal{W}$, on peut supposer ${ }^{6}$ que pour tout difféomorphisme $\varphi \in \mathcal{W}$, et tout $\lambda<1$, le difféomorphisme $x \mapsto \lambda \varphi\left(\lambda^{-1} x\right)$ est encore dans $\mathcal{W}$.

Il existe une décomposition de $\mathbb{R}^{2 d}$ en somme de trois espaces invariants $\mathbb{R}^{d}=E^{c} \oplus E^{s} \oplus E^{u}$, de façon que $E^{c}, E^{s}$ et $E^{u}$ correspondent aux valeurs propres de $L$ de module $1,<1$ et $>1$, respectivement. Nous construisons indépendamment pour $E^{c}, E^{s}$ et $E^{u}$ des familles de cubes quadrillés $\mathcal{C}^{c}, \mathcal{C}^{s}$ et $\mathcal{C}^{u}$, respectivement ${ }^{7}$. Nous en déduirons, à la fin de cette preuve, une famille $\mathcal{C}$ vérifiant les propriétés de la définition 4.3 en considérant des cubes homothétiques à ceux des familles ci-dessus.

Nous construisons d'abord la famille $\mathcal{C}^{c}$. La restriction de $L$ à $E^{c}$ est complètement elliptique et sans résonnance non triviale. Quitte à changer de coordonnées, les sphères $K_{r}$ de rayon $r$ de $E^{c}$ sont invariantes par $L$. La dynamique de $L$ sur $K_{r}$ est transitive par chaînes.

Comme dans la démonstration de la proposition 4.13, il existe une famille finie $\mathcal{C}^{c}$ de cubes quadrillés de $\left(L, \mathcal{W}, N_{0}+k\right)$ dont les adhérences des supports sont deux à deux disjointes, et qui est couvrante pour $K_{1}$. Il existe également un nombre $\delta^{c}>0$, un entier $m^{c}>0$ et une famille $\mathcal{D}^{c}$ de compacts contenus dans l'intérieur des carreaux des cubes de $\mathcal{C}^{c}$ tels que toute $\delta^{c}$-pseudo-orbite de longueur supérieure à $m^{c}$ et possédant un point $\delta^{c}$-proche de $K_{1}$ rencontre un compact de $\mathcal{D}^{c}$. De plus, pour tous compacts $D_{e}$ et $D_{s}$ de $\mathcal{D}^{c}$, il existe une pseudo-orbite de $K_{1}$, à sauts dans l'intérieur des carreaux des cubes de $\mathcal{C}^{c}$, joignant un point de $D^{e}$ à un point de $D^{s}$. Remarquons que l'un des compacts au moins de la famille $\mathcal{D}^{c}$ rencontre $K_{1}$; quitte à augmenter légèrement ce compact on peut supposer qu'il rencontre $K_{1}$ dans son intérieur.

Par notre choix de $\mathcal{W}$, si $C$ est un cube quadrillé pour $\mathcal{W}$, alors, pour tout $r \in] 0,1$ [, le cube homothétique $r \cdot C$ est encore un cube quadrillé pour $\mathcal{W}$. On notera $\mathcal{C}_{r}^{c}$ (resp. $\mathcal{D}_{r}^{c}$ ) la famille obtenue en multipliant les cubes de $\mathcal{C}^{c}$ (resp. les carreaux de $\mathcal{D}^{c}$ ) par $r$. La famille $\mathcal{C}_{r}^{c}$ est une famille couvrante de $K_{r}$ pour $\left(L, \mathcal{W}, N_{0}+k\right)$. Les familles $\mathcal{C}_{r}^{c}$ et $\mathcal{D}_{r}^{c}$ vérifient les mêmes propriétés que $\mathcal{C}^{c}$ et $\mathcal{D}^{c}$ avec la constante $r \delta^{c}$.

Construisons à présent la famille $\mathcal{C}^{s}$. Nous considérons un domaine fondamental compact $\Delta^{s}$ de $E^{s}$ : toute orbite de $E^{s} \backslash\{0\}$ pour $L$ rencontre $\Delta^{s}$ et $\Delta^{s} \cap L^{i}\left(\Delta^{s}\right)$ est vide dès que $|i|>1$.

6. Tout $C^{1}$-voisinage $\mathcal{W}_{0}$ de l'identité pour la topologie forte de Whitney contient un voisinage $\mathcal{W}$ qui a cette propriété : en effet, on peut chosir $\mathcal{W}$ défini par $f \in \mathcal{W}$ si $\|f(x)-x\|+\|D f(x)-i d\|<\psi(\|x\|)$ où $\psi$ est une fonction décroissante. Ce type d'ouvert forme une base de voisinage de l'identité.

7. Les cubes auront la dimension de la variété ambiante, contrairement à ce que pourraît laisser supposer la notation. 
Il existe alors $\varepsilon>0$ tel que pour toutes boules $B_{1}, B_{2}$ de rayon $\varepsilon$ centrées en des points de $\Delta^{s}$, et tous entiers $n_{1}, n_{2}$, l'intersection $L^{n_{1}}\left(B_{1}\right) \cap L^{n_{2}}\left(B_{2}\right)$ est vide dès que $\left|n_{1}-n_{2}\right|$ est strictement plus grand que 1 . On couvre alors $\Delta^{s}$ par un nombre fini de boules $B_{i}$ de rayon $\varepsilon$ centrées sur des points de $\Delta^{s}$. On choisit des entiers $n_{i}$ tels $\left|n_{i}-n_{j}\right|>100 d\left(N_{0}+k\right)$ si $i \neq j$. L'ouvert $U^{s}=\bigcup_{i} L^{n_{i}}\left(B_{i}\right)$ est un ouvert disjoint de ses $10 \cdot(2 d) \cdot\left(N_{0}+k\right)$ premiers itérés et rencontrant toute orbite de $\Delta^{s}$. La remarque 4.9 assure l'existence d'une famille finie $\mathcal{C}^{s}$ de cubes quadrillés de $\left(L, \mathcal{W}, N_{0}+k\right)$ dont les adhérences des supports sont deux à deux disjointes, et une famille $\tilde{\mathcal{D}}^{s}$ de compacts contenus dans des intérieurs de carreaux de cubes de $\mathcal{C}^{s}$, tels que toute orbite de $\Delta^{s}$ (et donc de $E^{s} \backslash\{0\}$ ) rencontre l'intérieur d'un de ces compacts. On peut donc supposer que chaque compact de $\tilde{\mathcal{D}}^{s}$ rencontre $E^{s}$ dans son intérieur (il suffit pour cela de restreindre la famille $\tilde{\mathcal{D}}^{s}$ à ceux qui possèdent cette propriété). Pour chacun des compacts $\tilde{D} \in \tilde{\mathcal{D}}^{s}$ on choisit un compact $D$ inclus dans l'intérieur du même carreau et contenant $\tilde{D}$ dans son intérieur. On note $\mathcal{D}^{s}$ la famille des compacts $D$ ainsi obtenus. On contruira de façon analogue les familles $\mathcal{C}^{u}, \mathcal{D}^{u}$ et $\tilde{\mathcal{D}}^{u}$.

Pour tout $r \leq 1$, on définit les familles $\mathcal{C}_{r}^{s}, \mathcal{D}_{r}^{s}, \tilde{\mathcal{D}}_{r}^{s}, \mathcal{C}_{r}^{u}, \mathcal{D}_{r}^{u}$ et $\tilde{\mathcal{D}}_{r}^{u}$ images des familles $\mathcal{C}^{s}$, $\mathcal{D}^{s}, \tilde{\mathcal{D}}^{s}, \mathcal{C}^{u}, \mathcal{D}^{u}$ et $\tilde{\mathcal{D}}^{u}$ par l'homothétie de rapport $r$ : ce sont des familles de cubes quadrillés et de compacts contenus dans l'intérieurs des carreaux des cubes, vérifiants toutes les propriétés énoncées ci-dessus.

La fin de la démonstration de la proposition 4.16 découle de la proposition suivante :

Proposition 4.18. Avec les notations ci-dessus, il existe $r \leq 1, t \leq 1$, il existe des voisinages $W \subset V$ de 0 et une constante $\delta>0$ tels que les familles $\mathcal{C}=\mathcal{C}^{s} \cup \mathcal{C}_{r}^{u} \cup \mathcal{C}_{t}^{c}$ (de cubes quadrillés pour $\left(L, \mathcal{W}, N_{0}\right)$ ) et $\mathcal{D}=\mathcal{D}^{s} \cup \mathcal{D}_{r}^{u} \cup \mathcal{D}_{t}^{c}$ (de compacts dans l'intérieur des carreaux) vérifient la définition 4.3.

Démonstration : Soit $V$ une boule ouverte contenant 0 et les adhérences des supports des cubes des familles $\mathcal{C}^{s}, \mathcal{C}^{u}$ et $\mathcal{C}^{c}$. L'application $L$ étant linéaire, produit d'une contraction de $E^{s}$, d'une dilatation de $E^{u}$ et d'une isométrie de $E^{c}$, on peut choisir $V$ de façon que si $x \in V$ et $L(x) \notin V$ (resp. $\left.L^{-1}(x) \notin V\right)$ alors pour tout $n>0$ on a $L^{n}(x) \notin V\left(\right.$ resp. $\left.L^{-n}(x) \notin V\right)$.

On choisira $r$ assez petit, puis $t$ assez petit pour que les adhérences des supports des cubes (considérés comme cubes quadrillés pour $\left(L, \mathcal{W}, N_{0}+k\right)$ ) de $\mathcal{C}=\mathcal{C}^{s} \cup \mathcal{C}_{r}^{u} \cup \mathcal{C}_{t}^{c}$ soient deux à deux disjointes. Ceci entraînera les items 1, 2, 3 et 6 de la définition 4.3. Désormais $r$ sera supposé fixé (assez petit); le choix de $t$ dépend encore des constructions suivantes.

Les familles de compacts $\mathcal{D}^{s}$ et $\mathcal{D}_{r}^{u}$ sont donc à présent fixées. On notera $\mathbb{D}^{s}$ l'union des compacts de la famille $\tilde{\mathcal{D}}^{s}$ et de même on notera $\mathbb{D}^{u}=\bigcup_{\tilde{D} \in \tilde{\mathcal{D}}_{r}^{u}} \tilde{D}$.

Lemme 4.19. L'union $\left(E^{u} \oplus E^{c}\right) \cup \bigcup_{n>0} L^{n}\left(\mathbb{D}^{s}\right)$ contient un voisinage $U^{s}$ de 0 . De même $L^{\prime}$ union $\left(E^{s} \oplus E^{c}\right) \cup \bigcup_{n \geq 0} L^{-n}\left(\mathbb{D}^{u}\right)$ contient un voisinage $U^{u}$ de 0 .

Démonstration : L'union des itérés positifs de $\mathbb{D}^{s}$ contient par construction un voisinage tubulaire d'un domaine fondamental compact $Z^{s}$ de $E^{s} \backslash\{0\}$, de la forme $Z^{s}+B^{c}+B^{u}$ où $B^{c}$ et $B^{u}$ sont des boules centrées en 0 dans $E^{c}$ et $E^{u}$, respectivement. En choisissant cette boule $B^{c}$ dans de bonnes coordonnées, elle est invariante par $L$. De même, on peut choisir $B^{u}$ pour que $L\left(B^{u}\right)$ contienne $B^{u}$. On en déduit que l'union des itérés positifs de $\mathbb{D}^{s}$ et de $B^{s}+B^{u}$ contient un voisinage de 0 de la forme $B^{s}+B^{c}+B^{u}$, où $B^{s}$ est un boule centrée en 0 dans $E^{s}$.

Remarque 4.20. Il existe une boule $B^{c} \subset E^{c}$, centrée en 0 , telle que, pour tout compact $\tilde{D}$ de $\tilde{\mathcal{D}}^{s}$ (resp. $\tilde{\mathcal{D}}_{r}^{u}$ ) l'union des itérés positifs (resp. négatifs) de $\tilde{D}$ contient $B^{c}$ dans son adhérence. 
On note $U$ une boule de $\mathbb{R}^{2 d}$, centrée en 0 et d'adhérence contenue dans $U^{s} \cap U^{u} \cap V$. On choisit $t$ tel que les adhérences des supports des cubes de la famille $\mathcal{C}_{t}^{c}$ ainsi que la sphère $K_{t} \subset E^{c}$ soient contenus dans $U$. Quitte à diminuer $t$ on peut supposer que $K_{t}$ est incluse dans $B^{c}$ (definie ci-dessus).

Pour tout $\eta>0$ on note $T_{\eta}$ le $\eta$-voisinage de $E^{c}$.

Lemme 4.21. Il existe $\eta_{0}, \delta_{0}>0$ et un voisinage $W_{0}$ de 0 tel que :

- toute $\delta_{0}$-pseudo-orbite $\left\{x_{0}, x_{1}, \ldots, x_{k}\right\}$ vérifiant $x_{0} \notin U, x_{k} \in W_{0}$ et $\left\{x_{1}, \ldots, x_{k-1}\right\} \subset T_{\eta_{0}}$, possède un point dans un compact de $\mathcal{D}_{t}^{c}$;

- toute $\delta_{0}$-pseudo-orbite $\left\{x_{0}, x_{1}, \ldots, x_{k}\right\}$ vérifiant $x_{0} \in W_{0}, x_{k} \notin U$ et $\left\{x_{1}, \ldots, x_{k-1}\right\} \subset T_{\eta_{0}}$, possède un point dans un compact de $\mathcal{D}_{t}^{c}$.

Démonstration : Par le choix de la famille $\mathcal{D}^{c}$, il suffit de voir qu'une telle pseudo-orbite pour $\delta_{0}$ assez petit (en particulier inférieur à $t \delta^{c}$ ), possède un point $t \delta^{c}$ proche de $K_{t}$ et est de longueur supérieure à $m^{c}$.

Notons $r_{i}$ la norme de la projection sur $E^{c}$ parallèle à $E^{s}+E^{u}$, du point $x_{i}$. Pour $\eta_{0}$ et $\delta_{0}$ assez petits, la différence $\left|r_{i}-r_{i+1}\right|$ sera arbitrairement petite. En supposant $x_{0} \notin U$ et $x_{k} \in W_{0}$, on vérifie $r_{0}>t$, car $U$ contient un voisinage de la boule bordée par $K_{t}$ dans $E^{c}$. De plus, pour $W_{0}$ choisi assez petit, $r_{k}$ sera plus petit que $t$. Il existe donc $i>0$ tel que $r_{i} \geq t$ et $r_{i+1}<t$. Le point $x_{i}$ appartient à $T_{\eta_{0}}$ et sa projection sur $E^{c}$ est arbitrairement proche de $K_{t}$. De ce fait (pour $\eta_{0}$ assez petit) il est $t \delta^{c}$ proche de $K_{t}$. Finalement, pour $W_{0}$ et $\delta_{0}$ assez petits, toute $\delta_{0}$ pseudo-orbite joignant le complémentaire de $U$ à $W_{0}$ est de longueur supérieure à $m^{c}$.

Le cas $x_{0} \in W_{0}$ et $x_{k} \notin U$ se traite de la même façon.

Lemme 4.22. Pour tout $\eta>0$, il existe $\delta_{\eta}>0$ et $m_{\eta} \geq 1$ tels que, pour toute $\delta_{\eta}$-pseudo-orbite $\left(x_{-m_{\eta}}, \ldots, x_{m_{\eta}}\right)$ de $\mathbb{R}^{2 d}$ telle que $x_{0}$ appartient à $U \backslash T_{\eta}$, il existe un entier $k \in\left\{0, \ldots, m_{\eta}\right\}$ tel que :

- ou bien $x_{k}$ appartient à compact de $\mathcal{D}^{u}$ et le segment de pseudo-orbite $\left\{x_{0}, \ldots, x_{k}\right\}$ est contenu dans $V$

- ou bien $x_{-k}$ appartient à un compact de $\mathcal{D}^{s}$ et le segment de pseudo-orbite $\left\{x_{-k}, \ldots, x_{0}\right\}$ est contenu dans $V$.

Démonstration : D'après le lemme 4.19 tout point de $U^{s} \cap U^{u} \backslash E^{c}$ a un itéré positif dans $\mathbb{D}^{u}$ ou un itéré négatif dans $\mathbb{D}^{s}$. L'adhérence de $U \backslash T_{\eta}$ est un compact inclus dans $U^{s} \cap U^{u} \backslash E^{c}$. Il existe donc $m_{\eta} \geq 1$ tel que tout point $x \in U \backslash T_{\eta}$ a un itéré positif $L^{k}(x)$ avec $k \in\left\{0, \ldots, m_{\eta}\right\}$ qui appartient à l'intérieur d'un compact de $\mathcal{D}^{u}$ ou un itéré négatif $L^{-k}(x)$ avec $k \in\left\{0, \ldots, m_{\eta}\right\}$ qui appartient à l'intérieur d'un compact de $\mathcal{D}^{s}$. De plus, par le choix de la boule $V$, comme $x$ et $L^{k}(x)$ (resp. $\left.L^{-k}(x)\right)$ appartiennent à $V$, il en va de même pour les points intermédiaires.

Pour $\delta_{0}$ assez petit, cette propriété reste valable pour les $\delta_{0}$-pseudo-orbites.

On fixe $\eta$ inférieur à $t . \delta_{t}^{c}$ et à la constante $\eta_{0}$ du lemme 4.21. On considère les constantes $\delta_{\eta}$ et $m=m_{\eta}$ correspondantes données par le lemme 4.22 ci-dessus. On introduit $\delta<\inf \left(\delta_{\eta}, \delta_{0}\right)$. On considère un voisinage $W \subset U \cap W_{0}$ de 0 dans $\mathbb{R}^{2 d}$. Si l'on choisit $W$ et $\delta$ suffisamment petits, toute $\delta$-pseudo-orbite de longueur $m$ et rencontrant $W$, est entièrement contenue dans $U \cap T_{\eta}$.

Soit $\left(x_{0}, \ldots, x_{\ell}\right)$ une $\delta$-pseudo-orbite de $\mathbb{R}^{2 d}$ pour $L$ qui joint le complémentaire de $V$ à $W$. Soit $j \in\{0, \ldots, \ell\}$ le plus petit entier tel que $\left\{x_{j}, \ldots, x_{\ell}\right\}$ est contenu dans $U \cap T_{\eta}$. En particulier 
$x_{j-1}$ n'appartient pas à $U \cap T_{\eta}$. D'après le choix de $\delta$ et de $W$ ceci implique que $\ell-j$ est supérieur à $m$.

Supposons d'abord que $x_{j-1}$ appartienne à $U$ (et donc à $U \backslash T_{\eta}$ ). Complétons la pseudo-orbite $\left\{x_{0}, \ldots, x_{\ell}\right\}$ par les points $x_{-n}=L^{-n}\left(x_{0}\right)$ pour tout entier positif $n$. Remarquons que tous les points $x_{j-1+k}$ avec $k \in\{0, \ldots, m\}$ sont bien définis et disjoints de $\mathbb{D}^{u}$ (car contenus dans $T_{\eta} \cap U$ ). D'après le lemme 4.22 , il existe un entier $k \in\{0, \ldots, m\}$ tel que $x_{j-1-k}$ appartienne à $\mathbb{D}^{s}$ et tel que la suite $\left\{x_{j-1-k}, \ldots, x_{j-1}\right\}$ est incluse dans $V$. En particulier, $j-1-k>0\left(\operatorname{car} x_{0} \notin V\right)$. Nous avons montré que dans ce cas, la pseudo-orbite $\left\{x_{0}, \ldots, x_{\ell}\right\}$ rencontre un des compacts de $\mathcal{D}^{s}$.

Si $x_{j-1}$ n'appartient pas à $U$, alors le lemme 4.21 montre que la pseudo-orbite $\left\{x_{j-1}, \ldots, x_{\ell}\right\}$ rencontre un des compacts de $\mathcal{D}_{t}^{c}$.

On montre de la même façon que toute $\delta$-pseudo-orbite joignant un point de $W$ au complémentaire de $V$ rencontre un compact de $\mathcal{D}_{r}^{u} \cup \mathcal{D}_{t}^{c}$.

Les familles $\mathcal{D}_{e}=\mathcal{D}^{s} \cup \mathcal{D}_{t}^{c}$ et $\mathcal{D}_{s}=\mathcal{D}_{r}^{u} \cup \mathcal{D}_{t}^{c}$ vérifient donc l'item 4 de la définition 4.3.

Il nous reste à montrer que les familles $\mathcal{D}_{e}$ et $\mathcal{D}_{s}$ vérifient l'item 5 . Considérons $D_{e} \in \mathcal{D}_{e}$ et $D_{s} \in \mathcal{D}_{s}$. Nous examinons les différents cas suivants :

- Si $D_{e} \in \mathcal{D}^{s}$ et $D_{s} \in \mathcal{D}_{r}^{u}$, alors $D_{e}$ et $D_{s}$ rencontrent dans leurs intérieurs respectifs les espaces stable $\left(E^{s}\right)$ et instable $\left(E^{u}\right)$ de $L$. Un itéré positif de $D_{e}$ rencontre donc $D_{s}$.

- Si $D_{e}$ et $D_{s}$ appartiennent tous deux à $\mathcal{D}_{t}^{c}$, alors, par définition de $\mathcal{D}^{c}$, il existe une pseudoorbite de $K_{t}$ à sauts dans l'intérieur des carreaux des cubes de $\mathcal{C}_{t}^{c}$, joignant un point de $D_{e}$ à un point de $D_{s}$.

- Si $D_{e} \in \mathcal{D}^{s}$ et $D_{s} \in \mathcal{D}_{t}^{c}$; alors il existe $D^{\prime} \in \mathcal{D}_{t}^{c}$ contenant dans son intérieur un point $z$ de $K_{t}$. Le choix de $t$ (voir aussi la remarque 4.20) montre qu'un itéré positif de $D_{e}$ contient des points arbitrairement proches de $z$ et donc dans $D^{\prime}$. D'autre part il existe, par l'item ci-dessus, une pseudo-orbite de $K_{t}$ à sauts dans l'intérieur des carreaux de des cubes de $\mathcal{C}_{t}^{c}$ joignant $D^{\prime}$ à $D_{s}$. En concaténant ces deux pseudo-orbites, on obtient une pseudo-orbite, à sauts dans l'intérieur des carreaux de $\mathcal{D}$, qui joint $D_{e}$ à $D_{s}$.

- Le cas $D_{e} \in \mathcal{D}_{t}^{c}$ et $D_{s} \in \mathcal{D}_{r}^{u}$ est similaire au cas précédent.

Ceci conclut la preuve de la proposition 4.18 et donc de la proposition 4.16.

\section{Démonstration des résultats principaux}

Puisque l'espace des difféomorphismes symplectiques d'une variété symplectique compacte vérifie l'axiome de perturbation locale, les théorèmes 2 et 4 sont des conséquences directes du théorème 6 et du théorème 7 (ou plus précisément de la proposition 4.2).

\subsection{Résultats de généricité dans le cas compact}

Démonstration du théorème 1 : D'après le théorème 8 , il existe un $G_{\delta}$ dense $\mathcal{G}_{0} \operatorname{de} \operatorname{Diff}_{\omega}^{1}(M)$ tel que les orbites périodiques de tout élément de $\mathcal{G}_{0}$ n'ont pas de résonance non triviale. Soit alors $\left(U_{n}\right)$ une base dénombrable d'ouverts non vides de $M$. On définit pour tout couple $(n, m)$ d'entiers la propriété $\mathcal{P}_{n, m}$ par : $f$ vérifie $\mathcal{P}_{n, m}$ si $\bigcup_{k \geq 1} f^{k}\left(U_{n}\right) \cap U_{m} \neq \emptyset$. L'ensemble $\mathcal{U}_{m, n}$ des éléments de $\operatorname{Diff}_{\omega}^{1}(M)$ qui vérifient $\mathcal{P}_{m, n}$ est clairement ouvert, donc $\mathcal{V}_{m, n}=\mathcal{U}_{m, n} \cup\left(\overline{\mathcal{U}_{m, n}}\right)^{c}$ est 
un ouvert dense de $\operatorname{Diff}_{\omega}^{1}(M)$, donc $\mathcal{G}=\mathcal{G}_{0} \cap \bigcap_{(m, n)} \mathcal{V}_{m, n}$ est un $G_{\delta}$ dense de $\operatorname{Diff}_{\omega}^{1}(M)$. Considérons alors $f \in \mathcal{G}$, et deux entiers $m$ et $n$. Supposons que $f \in\left(\overline{\mathcal{U}_{m, n}}\right)^{c}$; soient alors $x \in U_{n}$ et $y \in U_{m}$; alors $x \dashv y$, donc par le théorème 2, il existe $g \in\left(\overline{\mathcal{U}_{m, n}}\right)^{c}$ tel que $y$ est sur l'orbite strictement positive de $x$, donc $g \in\left(\overline{\mathcal{U}_{m, n}}\right)^{c} \cap \mathcal{U}_{m, n}$, ce qui est impossible. Donc finalement $f \in \mathcal{U}_{m, n}$, et donc $f$ est transitif. Ceci montre que l'ensemble des difféomorphismes transitifs de $\operatorname{Diff}_{\omega}^{1}(M)$ contient un $G_{\delta}$-dense. D'autre part, cet ensemble coïncide avec $\bigcap_{(m, n)} \mathcal{U}_{m, n}$ qui est un $G_{\delta}$.

Dans [N], S. Newhouse montre que l'ensemble $\mathcal{G}_{1}$ des difféomorphismes symplectiques d'une variété compacte qui ont un ensemble dense de points périodiques hyperboliques est un $G_{\delta}$ dense de $\operatorname{Diff}_{\omega}^{1}(M)$. Soient alors $f \in \mathcal{G} \cap \mathcal{G}_{1}, \mathcal{U}$ un voisinage ouvert de $f$ dans $\operatorname{Diff}_{\omega}^{1}(M)$, et $p_{f}$ un point périodique hyperbolique de $f$; quitte à restreindre $\mathcal{U}$, on peut supposer que tout $g \in \mathcal{U}$ a un point hyperbolique $p_{g}$ proche de $p_{f}$, l'application $\left(g \mapsto p_{g}\right)$ étant continue. On va alors montrer qu'il existe un $G_{\delta}$-dense $\mathcal{G}_{\mathcal{U}}$ de $\mathcal{U}$ tel que la classe homocline de $p_{g}$ pour tout élément $g \in \mathcal{G}_{\mathcal{U}}$ est dense dans $M$. Cela terminera la démonstration. Pour cela, on définit pour chaque entier $n$ l'ensemble $\mathcal{W}_{n}$ des éléments $g$ de $\mathcal{U}$ tels que $W^{u}\left(O\left(p_{g}\right), g\right)$ et $W^{s}\left(O\left(p_{g}\right), g\right)$ rencontrent $U_{n}$. Chaque $\mathcal{W}_{n}$ est un ouvert de $\mathcal{U}$, et il existe une partie dense $\mathcal{G}_{\mathcal{U}}$ de $\mathcal{U}$ de difféomorphismes transitifs. Alors, si $g \in \mathcal{G}_{\mathcal{U}}$, on a pour $g$ : pour tout voisinage $V$ de $p_{g}$, il existe des itérés positifs $f^{i}(V)$ et négatifs $f^{-j}(V), i, j \geq 1$, qui rencontrent $U_{n}$. À l'aide du connecting lemma de S. Hayashi ${ }^{8}$, on en déduit qu'on peut trouver $h C^{1}$-proche de $g$, donc dans $\mathcal{U}$ qui est dans $\mathcal{W}_{n}$. Aussi, chaque $\mathcal{W}_{n}$ est dense, donc l'intersection $\mathcal{W}$ des $\mathcal{W}_{n}$ est un $G_{\delta}$ dense de $\mathcal{U}$, c'est-à-dire l'ensemble des éléments $g$ de $\mathcal{U}$ tels que la variété stable et la variété instable de $O\left(p_{g}\right)$ sont denses dans $M$ est un $G_{\delta}$ dense $\mathcal{W}$ de $\mathcal{U}$. Si maintenant $g \in \mathcal{G} \cap \mathcal{W}$, et si $n$ est un entier, tout point de $U_{n}$ est un point d'accumulation de $W^{u}\left(O\left(p_{g}\right), g\right)$ et $W^{s}\left(O\left(p_{g}\right), g\right)$; utilisant alors un tel point qui est non périodique, on peut, à l'aide du connecting lemma créer une intersection homocline dans $U_{n}$, qu'on peut rendre transverse par perturbation. Le fait que $p_{g}$ ait une intersection homocline transverse dans $U_{n}$ est alors une propriété ouverte et dense dans $\mathcal{U}$, ce qui permet de conclure : dans $\mathcal{U}$ l'ensemble des difféomorphismes $g$ pour lesquels la classe homocline de $p_{g}$ est la variété $M$ contient un $G_{\delta}$ dense (c'est même un $G_{\delta}$ par semi-continuité inférieure de la classe homocline). Ceci étant valable pour tout petit voisinage ouvert $\mathcal{U}$ d'éléments $f$ de $\mathcal{G} \cap \mathcal{G}_{1}$, on obtient que l'ensemble des difféomorphismes tels que la variété $M$ soit la classe homocline d'un point hyperbolique contient un $G_{\delta}$ dense dans $\operatorname{Diff}_{\omega}^{1}(M)$.

\section{Démonstration du corollaire 1.2 :}

On avait démontré dans $\left[\mathrm{A}_{2}\right]$ :

Théorème 9 (M.-C. Arnaud). Soit $(M, \omega)$ une variété compacte connexe symplectique de dimension 4. Il existe un $G_{\delta}$ dense $\mathcal{G}$ de $\operatorname{Diff}_{\omega}^{1}(M)$ tel que tout difféomorphisme $f$ de $\mathcal{G}$ vérifie une et une seule des trois propriétés suivantes :

- $f$ est Anosov;

- $f$ est partiellement hyperbolique avec une direction centrale de dimension 2.

- $f$ au moins une orbite périodique complètement elliptique.

Le théorème 1 nous permet de supposer de plus que tout $f \in \mathcal{G}$ est transitif. De plus, S. Newhouse a démontré dans $[\mathrm{N}]$ :

8. Le connecting lemma permet de construire une perturbation $g_{1} \in \mathcal{U}$ pour laquelle $W^{u}\left(O\left(p_{g_{1}}\right), g_{1}\right)$ rencontre $U_{n}$. On approche $g_{1}$ par un difféomorphisme $g_{2} \in \mathcal{G}_{\mathcal{U}}$. En appliquant à nouveau le connecting lemma, on obtient une perturbation $h \in \mathcal{U}$ de $g_{2}$ pour laquelle les variétés stables et instables de l'orbite de $p_{h}$ rencontrent $U_{n}$. 
Théorème 10 (S. Newhouse). Soit $(M, \omega)$ une variété symplectique compacte connexe. Il existe un $G_{\delta}$ dense $\mathcal{G}$ de $\operatorname{Diff}_{\omega}^{1}(M)$ tel que si $f \in \mathcal{G}$, soit $f$ est Anosov, soit l'ensemble des points périodiques elliptiques de $f$ est dense dans $M$.

On supposera donc que tout $f \in \mathcal{G}$ vérifie aussi cette propriété.

Soit $U_{1}$ l'ensemble des difféomorphismes d'Anosov de $\operatorname{Diff}_{\omega}^{1}(M)$ et $U_{2}$ l'ensemble de ceux qui sont partiellement hyperboliques avec une direction centrale de dimension 2.

Soit $f \in \mathcal{G}$. Supposons que $f$ n'est pas dans $\bar{U}_{1}$. Alors, par le théorème 10 , il existe une suite $\left(p_{n}\right)$ de points périodiques elliptiques qui forment une partie dense de $M$. Comme cette partie est dense dans $M$ et que $f$ est transitif, on peut supposer, quitte à extraire une sous-suite, que la suite des orbites $O\left(p_{n}\right)$ converge vers $M$ pour la topologie de Hausdorff : en effet une orbite périodique elliptique très proche d'un point $x$ d'orbite dense sera $\varepsilon$-dense pour un $\varepsilon$ arbitraire.

Aussi, si $f \in \mathcal{G} \cap U_{2}, f$ a une suite d'orbites périodiques elliptiques ayant un espace central de dimension 2 (correspondant à $E_{c}$ ) qui converge vers $M$ pour la topologie de Hausdorff. De plus, on sait que $U_{2}$ est ouvert dans $\operatorname{Diff}_{\omega}^{1}(M)$ et [DW] montre qu'il contient un ouvert dense $\mathcal{P}$ de difféomorphismes robustement transitifs. Alors, tout $f \in \mathcal{G} \cap \mathcal{P}$ (où $\mathcal{G} \cap \mathcal{P}$ est un $G_{\delta}$ dense de $U_{2}$ ) est partiellement hyperbolique, robustement transitif, et a une suite d'orbites périodiques elliptiques ayant un espace central de dimension 2 correspondant à $E_{c}$ qui converge vers $M$ en topologie de Hausdorff.

Considérons l'ensemble $\mathcal{R}$ des difféomorphismes de $\operatorname{Diff}_{\omega}^{1}(M) \backslash\left(\bar{U}_{1} \cup \bar{U}_{2}\right)$ qui ont une suite d'orbites périodiques complètement elliptiques et dont les valeurs propres sont toutes de multiplicité 1 (en particulier sont différentes de 1), tendant vers $M$ en topologie de Hausdorff. On va montrer que $\mathcal{R}$ est un $G_{\delta}$ dense de $\operatorname{Diff}_{\omega}^{1}(M) \backslash\left(\bar{U}_{1} \cup \bar{U}_{2}\right)$. Ceci terminera la démonstration du corollaire.

Pour chaque $k \in \mathbb{N}^{*}$, notons $\mathcal{U}_{k}$ l'ensemble des difféomorphismes de $\operatorname{Diff}_{\omega}^{1}(M) \backslash\left(\bar{U}_{1} \cup \bar{U}_{2}\right)$ qui ont une orbite périodique complètement elliptique dont les valeurs propres sont toutes de multiplicité 1 qui est $\frac{1}{k}$-proche de $M$ pour la distance de Hausdorff. $\mathcal{U}_{k}$ est une partie ouverte de $\operatorname{Diff}_{\omega}^{1}(M) \backslash\left(\bar{U}_{1} \cup \bar{U}_{2}\right)$, l'intersection des $\mathcal{U}_{k}$ est $\mathcal{R}$, il nous suffit donc de montrer que chaque $\mathcal{U}_{k}$ est dense pour conclure.

Soient $\mathcal{V}$ un ouvert non vide de $\operatorname{Diff}_{\omega}^{1}(M) \backslash\left(\bar{U}_{1} \cup \bar{U}_{2}\right), f$ un élément de $\mathcal{V} \cap \mathcal{G} \cap \operatorname{Diff}_{\omega}^{1}(M) \backslash\left(\bar{U}_{1} \cup\right.$ $\left.\bar{U}_{2}\right)$. D'après le théorème 10 et la transitivité de $f$, il existe une suite $\left(p_{n}(f)\right)_{n \in \mathbb{N}}$ d'orbite périodiques elliptiques tendant vers $M$ pour la topologie de Hausdorff. Quitte à supprimer les premiers termes de la suite, on peut supposer que tous les $O\left(p_{n}(f)\right)$ sont à une distance de Hausdorff inférieure à $\frac{1}{k}$ de $M$. Si l'un de ces orbites $O\left(p_{N}(f)\right)$ est complètement elliptique, on peut trouver $g \in \mathcal{V}$ pour lequel $O\left(p_{N}(f)\right)$ est une orbite périodique de $g$ complètement elliptique et dont les valeurs propres sont de multiplicité 1 . On suppose désormais qu'aucune de ces orbites n'est complètement elliptique.

On utilise alors la proposition suivante :

Proposition 5.1. Soient $(M, \omega)$ une variété symplectique compacte de dimension 4 , et $f \in$ $\operatorname{Diff}_{\omega}^{1}(M)$. Soit $\mathcal{E} \subset M$ un ensemble d'orbites périodiques elliptiques non-complètement elliptiques de $f$. Si la décomposition naturelle (en espaces propres) de la restriction $\left.T M\right|_{\mathcal{E}}$ en $\left.T M\right|_{\mathcal{E}}=E^{s} \oplus E^{c} \oplus E^{u}$ n'est pas dominée alors, pour tout $C^{1}$-voisinage $\mathcal{U}$ de $f$ et tout $\varepsilon>0$, il existe $g \in \mathcal{U}$ et une orbite (de $f)\left\{x_{1}, \ldots, x_{k}\right\} \in \mathcal{E}$ et une orbite $\left\{y_{1}, \ldots, y_{\ell}\right\}$ de $g$ qui est complètement elliptique et telle que $\left\{x_{1}, \ldots, x_{k}\right\}$ est contenu dans l'E-voisinage de $\left\{y_{1}, \ldots, y_{\ell}\right\}$. 
Voyons comment conclure. Si la décomposition naturelle sur l'union des orbites $O\left(p_{N}(f)\right)$ est dominée, alors la décomposition s'étend à l'adhérence de cette union, c'est-à-dire à $M$ (d'après [BDP, Lemma 1.4]). Dans ce cas $f$ est partiellement hyperbolique, d'après la remarque 1.1, item 2. Puisque $f$ n'appartient pas à $\bar{U}_{1} \cup \bar{U}_{2}$, cette décomposition n'est pas dominée. La proposition 5.1 construit alors, par $C^{1}$-petite perturbation, des orbites complètement elliptiques qui sont $\frac{1}{k}$ denses dans $M$ : c'est ce qu'il nous restait à montrer.

La proposition 5.1 est l'équivalent de [ $\mathrm{A}_{2}$, Proposition 11]. Dans cette proposition, les hypothèses et la conclusion étaient légèrement différentes : $\mathcal{E}$ était l'ensemble des orbites périodiques elliptiques de $f$ et était supposé dense dans $M$, et $f$ ne possèdait aucun point complètement elliptique; la conclusion était que, si la décomposition naturelle (en espace propres) n'est pas dominée, alors une perturbation de $f$ possède un point(stablement) complètement elliptique. La preuve donnée par $\left[\mathrm{A}_{2}\right]$ consiste en fait à montrer l'énoncé de la proposition 5.1.

\subsection{Résultats de généricité dans le cas non compact}

Nous expliquons dans cette partie comment déduire du théorème 4 le théorème 3. Pour cela, nous allons avoir besoin d'une proposition, qui demande d'introduire une nouvelle relation entre les points de $M$. Celle-ci a été introduite dans $\left[\mathrm{A}_{1}\right]$ : si $f$ est un élément de $\operatorname{Diff}_{\omega}^{1}(M)$, si $x$ et $y$ sont deux points de $M$, on écrit $x \prec_{f} y$ ou $x \prec y$ quand, pour tous voisinages $U$ et $V$ de $x$ et $y$ respectivement, l'ensemble $\bigcup_{k \geq 1} f^{k}(U) \cap V$ n'est pas vide.

Proposition 5.2. Il existe un $G_{\delta}$ dense $\mathcal{G}$ de $\operatorname{Diff}_{\omega}^{1}(M)$ tel que : pour tout $f \in \mathcal{G}$, pour tout compact $K$ invariant par $f$, pour tous $x$, $y$ de $M$ tels que $x \dashv_{K} y$, alors $x \prec y$.

Démonstration de la proposition 5.2 : D'après le théorème 8 , il existe un $G_{\delta}$ dense $\mathcal{G}_{0}$ de $\operatorname{Diff}_{\omega}^{1}(M)$ tel que les orbites périodiques de tout élément de $\mathcal{G}_{0}$ n'ont pas de résonance non triviale. Soit alors $\left(U_{n}\right)$ une base dénombrable d'ouverts non vides de $M$. On définit, pour tout couple $(n, m)$ d'entiers, la propriété $\mathcal{P}_{n, m}$ par : $f$ vérifie $\mathcal{P}_{n, m}$ si $\bigcup_{k \geq 1} f^{k}\left(U_{n}\right) \cap U_{m} \neq \emptyset$. L'ensemble $\mathcal{U}_{m, n}$ des éléments de $\operatorname{Diff}_{\omega}^{1}(M)$ qui vérifient $\mathcal{P}_{m, n}$ est clairement ouvert. Ainsi $\mathcal{V}_{m, n}=\mathcal{U}_{m, n} \cup\left(\overline{\mathcal{U}_{m, n}}\right)^{c}$ est un ouvert dense de $\operatorname{Diff}_{\omega}^{1}(M)$, et $\mathcal{G}=\mathcal{G}_{0} \cap \bigcap_{(m, n)} \mathcal{V}_{m, n}$ est un $G_{\delta}$ dense de $\operatorname{Diff}_{\omega}^{1}(M)$. Considérons alors $f \in \mathcal{G}, K$ un compact de $M$ invariant par $f$ et $x$ et $y$ deux points de $M$ tels que $x \dashv_{K} y$. Montrons que $x \prec_{f} y$. Supposons que ce ne soit pas le cas. On peut alors trouver un voisinage $U_{n}$ de $x$, un voisinage $U_{m}$ de $y$ tels que $\bigcup_{k \geq 1} f^{k}\left(U_{n}\right) \cap U_{m}=\emptyset$. Aussi, $f \notin \mathcal{U}_{m, n}$. Comme $f \in \mathcal{G}$, forcément $f \in\left(\overline{\mathcal{U}_{m, n}}\right)^{c}$. Soit alors $\mathcal{V}$ un voisinage quelconque de $f$. Comme $x \dashv_{K} y$, on peut appliquer le théorème $4:$ si $x \neq y$ il existe $g \in \mathcal{V}$ tel que $y$ est sur l'orbite strictement positive de $x$ sous $g$, donc tel que $g \in \mathcal{U}_{m, n} ;$ si $x=y$, on applique le théorème aux points $x$ et $f^{-1}(y)$ : il existe alors $g \in \mathcal{V}$ et $\ell>0$ tel que $f^{-1}(y)=g^{\ell}(x)$ et donc $g^{\ell+1}\left(U_{n}\right) \cap U_{m} \neq \emptyset$. Ceci signifie que $f \in \overline{\mathcal{U}_{m, n}}$, ce qui contredit $f \in\left(\overline{\mathcal{U}_{m, n}}\right)^{c}$. Donc on a bien $x \prec_{f} y$.

Démonstration du théorème 3 : Il existe une suite $\left(K_{n}\right)_{n \in \mathbb{N}}$ de parties compactes de $M$ telles que pour tout $n \in \mathbb{N}$, on a $K_{n} \subset \operatorname{Int}\left(K_{n+1}\right)$ et $M=\bigcup_{n \in \mathbb{N}} K_{n}$. Soit $f \in \operatorname{Difff}_{\omega}^{1}(M)$. L'ensemble 
des points dont l'orbite positive est relativement compacte s'écrit :

$$
\mathcal{K}(f)=\bigcup_{n \in \mathbb{N}} \mathcal{K}_{n}(f) \quad \text { où } \quad \forall n \in \mathbb{N}, \quad \mathcal{K}_{n}(f)=\bigcap_{k \in \mathbb{N}} f^{-k}\left(K_{n}\right) .
$$

Chaque $\mathcal{K}_{n}(f)$ étant fermée, on sait par la propriété de Baire que si, pour tout $n \in \mathbb{N}$, l'intérieur de $\mathcal{K}_{n}(f)$ est vide, alors l'intérieur de $\mathcal{K}(f)$ est lui aussi vide. Définissons $\mathcal{K}_{n}^{\prime}(f)=\overline{\operatorname{Int}\left(\mathcal{K}_{n}(f)\right)}$. Comme $\mathcal{K}_{n}(f), \mathcal{K}_{n}^{\prime}(f)$ est un compact positivement invarariant par $f$ et a même intérieur que $\mathcal{K}_{n}(f)$. De plus, $\mathcal{K}_{n}^{\prime}(f)$ est l'adhérence de son intérieur. Pour montrer le théorème, il nous suffit de montrer que génériquement, $\mathcal{K}_{n}^{\prime}(f)$ est d'intérieur vide (donc est vide).

La proposition 5.2 nous fournit l'existence d'un $G_{\delta}$ dense $\mathcal{G}$ de $\operatorname{Diff}_{\omega}^{1}(M)$ tel que pour tout $f \in \mathcal{G}$, pour tout compact $K$ invariant par $f$, pour tous $x, y$ de $M$ tels que $x \dashv_{K} y$, alors $x \prec_{f} y$. Soit $f \in \mathcal{G}$. Supposons que pour un certain $n \in \mathbb{N}$, l'intérieur de $\mathcal{K}_{n}(f)$ soit non vide, et considérons une composante connexe $C$ (donc d'intérieur non vide) de $\mathcal{K}_{n}^{\prime}(f)$. Puisque le volume de $\mathcal{K}_{n}^{\prime}(f)$ est fini et que $f$ préserve le volume, le théorème de récurrence de Poincaré implique que $C$ est périodique. Notons $\tau$ sa période et $O(C)=\bigcup_{k=0}^{\tau-1} f^{k}(C)$ l'union des itérés de $C$.

Lemme 5.3. Avec les notations ci-dessus, la restriction de $f$ à $O(C)$ est transitive.

Démonstration : Rappelons que, si $U$ est un ouvert connexe contenu dans $\Omega(f)$, alors tous points $x, y \in U$ peuvent être joints par des pseudo-orbites, de sauts arbitrairement petits, contenues dans l'orbite positive de $U$ et à sauts dans $U$ (voir par exemple [BC, Lemme 5.1]).

Le théorème de récurrence de Poincaré implique que $C$ est inclus dans $\Omega(f)$, et $\operatorname{Int}(C)$ est connexe par construction. En conséquence, pour tous $x, y \in \operatorname{Int}(C)$ on a $x \dashv_{O(C)} y$. En utilisant la proposition 5.2 on obtient $x \prec_{f} y$. Comme $C$ est l'adhérence de son intérieur, ceci montre la transitivité de $f$ sur $O(C)$.

Soit $x \in \partial C$. Deux cas peuvent se produire :

1. Il existe un voisinage ouvert connexe $U$ de $x$ et un entier $p \geq n$ tel que $\bigcup_{k \in \mathbb{N}} f^{k}(U) \subset K_{p}$, c'est à dire $U \subset \mathcal{K}_{p}(f)$. En conséquence, $U \cup C$ est inclus dans une même composante connexe de $\mathcal{K}_{p}(f)$, et donc dans une même connexe $C^{\prime}$ de $\mathcal{K}_{p}^{\prime}(f)$. La restriction de $f$ à $O\left(C^{\prime}\right)$ est transitive d'après le lemme 5.3 ; aussi il existe une orbite joignant $\operatorname{Int}(C)$ à $U \backslash \mathcal{K}_{n}^{\prime}(f)$; ceci contredit l'invariance de $O(C)$ sous $f$.

2. Sinon, pour tout voisinage $O$ de $x$, il existe une orbite joignant $O$ à $M \backslash K_{n+1}$. En extrayant des sous-suites, on en déduit l'existence d'un point $y$ de $M \backslash \operatorname{Int}\left(K_{n+1}\right)$ tel que, pour tout voisinage $V$ de $y$ et tout voisinage $U$ de $x$, il existe une orbite joignant $U$ à $V$ : en d'autres termes, $x \prec_{f} y$. Aussi, par transitivité de $f$ sur $O(C)$, tout $z \in \operatorname{Int}(C)$ peut être joint à $y$ par une pseudo-orbite de sauts arbitrairement petits, et ayant au plus trois sauts : en $z$, en $x$ et en $y$. En conséquence, $z \dashv_{\mathcal{K}_{n}^{\prime}(f)} y$. D'après la proposition 5.2 on a $z \prec_{f} y$, ce qui contredit l'invariance de $\mathcal{K}_{n}^{\prime}(f)$ : comme $y \notin \operatorname{Int}\left(K_{n+1}\right)$ et $\mathcal{K}_{n}^{\prime}(f)$ est un compact contenu dans $\operatorname{Int}\left(K_{n+1}\right), y$ possède un voisinage $V$ disjoint de $\mathcal{K}_{n}^{\prime}(f)$; par ailleurs, $z$ possède un voisinage $U$ contenu dans $\mathcal{K}_{n}^{\prime}(f)$ et donc dont tous les itérés positifs sont disjoints de $V$.

\section{Références}

[A $\mathrm{A}_{1}$ M.-C. Arnaud, Création de connexions en topologie $C^{1}$, Ergod. Th. \& Dynam. Syst., 21, 339-381, (2001). 
$\left[\mathrm{A}_{2}\right] \quad$ M.-C. Arnaud, The generic symplectic $C^{1}$-diffeomorphisms of four-dimensional symplectic manifolds are hyperbolic, partially hyperbolic or have a completely elliptic periodic point, Ergod. Th. \& Dynam. Syst., 22, no. 6, 1621-1639, (2002).

[BV] J. Bochi et M. Viana, Lyapunov exponents : How frequently are dynamical systems hyperbolic?, prépublication IMPA, (2003).

[BC] Ch. Bonatti et S. Crovisier, Récurrence et généricité, Prépublication, (2002), à paraître à Invent. Math.

[BDP] Ch. Bonatti, L.J. Díaz et E.R. Pujals, $A \mathcal{C}^{1}$-generic dichotomy for diffeomorphisms : weak forms of hyperbolicicity or infinitely many sinks or sources, Ann. Math., 158, 355-418, (2003).

[DPU] L.J. Díaz, E.R. Pujals et R. Ures, Partial hyperbolicity and robust transitivity, Acta Mathematica, 183, 1-43, (1999).

[DW] D. Dolgopyat et A. Wilkinson, Stable accessibility is $C^{1}$-dense, Astérisque, 287, Geometric Methods in Dynamics (II), (Éditeurs : W. de Melo, M. Viana, J.-C. Yoccoz), 33-60, (2003).

[H] M. Herman, Sur les courbes invariantes par les difféomorphismes de l'anneau, Astérisque, $\mathbf{1 4 4},(1986)$.

[HT] V. Horita et A. Tahzibi, Robust Ergodicity and Dominated splittings for Symplectic diffeomorphisms, Conférence Robustness and partial hyperbolicity, Buzios (2003).

$[\mathrm{M}] \quad \mathrm{R}$. Mañé, The Lyapunov exponents of generic area preserving diffeomorphisms, International Conference on Dynamical Systems (Montevideo, 1995), 110-119, Pitman Res. Notes Math. Ser., 362, Longman, Harlow, (1996).

[N] S. Newhouse, Quasi-elliptic periodic points in conservative dynamical systems, Amer. J. of Math., 99, no 5, 1061-1087, (1977).

[OU] J. C. Oxtoby et S. M. Ulam, Measure-preserving homeomorphisms and metrical transitivity, Ann. of Math., 42, no 2, 874-920, (1941).

[PR] C. Pugh et C. Robinson, The $C^{1}$ closing lemma, including Hamiltonians, Erg. Th. \& Dyn. Syst., 3, 261-314, (1983).

[R] C. Robinson, Generic properties of conservative systems, Am. J. of Math., 92, 562-603, (1970).

[X] Z. Xia, Homoclinic points in symplectic and volume preserving diffeomorphisms, Comm. in Math. Phys, 177, 435-449, (1996).

[Y] J.-C. Yoccoz, Travaux de Herman sur les tores invariants, Séminaire Bourbaki 784, Astérisque, 206, 311-344, (1992).

Marie-Claude Arnaud (Marie-Claude.Arnaud@univ-avignon.fr)

Laboratoire d'Analyse non linéaire et de Géométrie, EA 2151

Faculté des sciences

33, rue louis Pasteur

84000 Avignon

France 
Christian Bonatti (bonatti@u-bourgogne.fr)

Sylvain Crovisier (Sylvain.Crovisier@u-bourgogne.fr)

Institut de Mathématiques de Bougogne, UMR 5584 du CNRS

BP 47870

21078 Dijon Cedex

France 